\title{
Die Froburgerstädte
}

Urs Wiesli

\section{Historischer Überblick}

Mit der Gründung Freiburgs i. Br. im Jahre 1120 begann nördlich der Alpen eine gewaltige Welle von Städtegründungen, die sich vom Beginn des 13 . Jahrhunderts an gleichsam zu einem Wetteifern im Städtegründen unter den großen und kleinen, weltlichen und geistlichen Herren steigerte. Diesem Zuge der Zeit folgten auch die Froburger Grafen, die eine Zeitlang zu den mächtigsten Geschlechtern im Gebiet von Jura und Mittelland gehörten. Versippt und verschwägert mit fast allen Hochadelsfamilien der Nachbarschaft, schienen sie im 12. und beginnenden 13. Jahrhundert im Begriff zu sein, ihre Herrschaft von der Gegend von Basel bis in den heutigen Kanton Luzern auszudehnen.

Die Stammburg der Froburger erhob sich auf 830 Metern Höhe auf einem Schuppenkamm des Juras $3 \mathrm{~km}$ nördlich von Olten, fast senkrecht über dem im Mittelalter wahrscheinlich noch öfters begangenen und schon den Römern bekannten $\mathrm{Paß}$ des Erlimooses, 778 Meter, und nur 1,5 km östlich der Paßhöhe des Untern Hauensteins, 691 Meter, eines Überganges also, der im Zusammenhang mit der Begehbarmachung des Gotthards beträchtlich an Bedeutung gewonnen hatte.

Kern des froburgischen Besitzes war die Landgrafschaft Buchsgau, ein Gebiet, das im Westen im Dünnertal begann, im Süden zwischen Siggermündung östlich von Solothurn und Erzbachmündung westlich Aarau durch die Aare begrenzt wurde und im Norden durch eine Linie bestimmt war, die etwa der Brandungszone zwischen Ketten- und Tafeljura folgt. Dazu kam die Grafschaft Sissgau, ungefähr identisch mit dem Einzugsgebiet der Ergolz und von der Brandungszone bis an den Rhein zwischen Birsmündung und Möhlin reichend. Drittes Gebiet war der Besitz im Aargau, ungefähr dem heutigen Bezirk Zofingen entsprechend (14).

Schon seit 1240 , mit der mehrfachen Teilung des froburgischen Besitzes, erfolgte ein rascher Zerfall des Geschlechtes. 1367 starb das Haus aus; Haupterben waren die Habsburger. Die Blütezeit der Froburger fällt also in die gleiche Zeit wie diejenige der Zähringer, die ebenfalls von den Habsburgern beerbt wurden. Schon 1356 wurde die Stammburg über dem Untern Hauenstein durch das große Basler Erdbeben zur Ruine. Seither ist sie nie wieder aufgebaut worden. 1
Die Lage der 1114 erstmals erwähnten Froburg ist Sinnbild für die enge Verknüpfung der Machtstellung des Geschlechts mit der Beherrschung der Jurapässe Unterer und Oberer Hauenstein. Die Sicherung des Gebietes erfolgte ursprünglich durch eine große Zahl von Burganlagen. Am Untern Hauenstein waren es vor allem die Wartenberge bei Muttenz und die Homburg bei Läufelfingen. Neben den Burgen gab es eine Reihe von Ministerialensitzen, so Wikon südlich Zofingen, die zwei Wartburgen südlich Olten, die Hagberg am nördlichen Stadtrand Oltens, Ifenthal und Itingen. Ähnlich am Obern Hauenstein: Hier standen die Waldenburg, das Schloß Bipp, die zwei Falkensteine, Bechburgen und Erlinsburgen in der Klusenlandschaft von Balsthal-Oensingen. Längs der Aare erhoben sich die Stützpunkte Fridau bei Murgenthal, die Aarburg, das Schloß Zielemp in Olten, Kienberg in der Aareschleife nördlich Olten, Winznau und Obergösgen. "... man kann sagen, daß jeder Teil des Froburger Besitzes bis zu den abgelegenen Juratälern durch Burgen gesichert war und daß fast bei jeder Ortschaft ein Dienstmannengeschlecht seinen Sitz hatte.»2

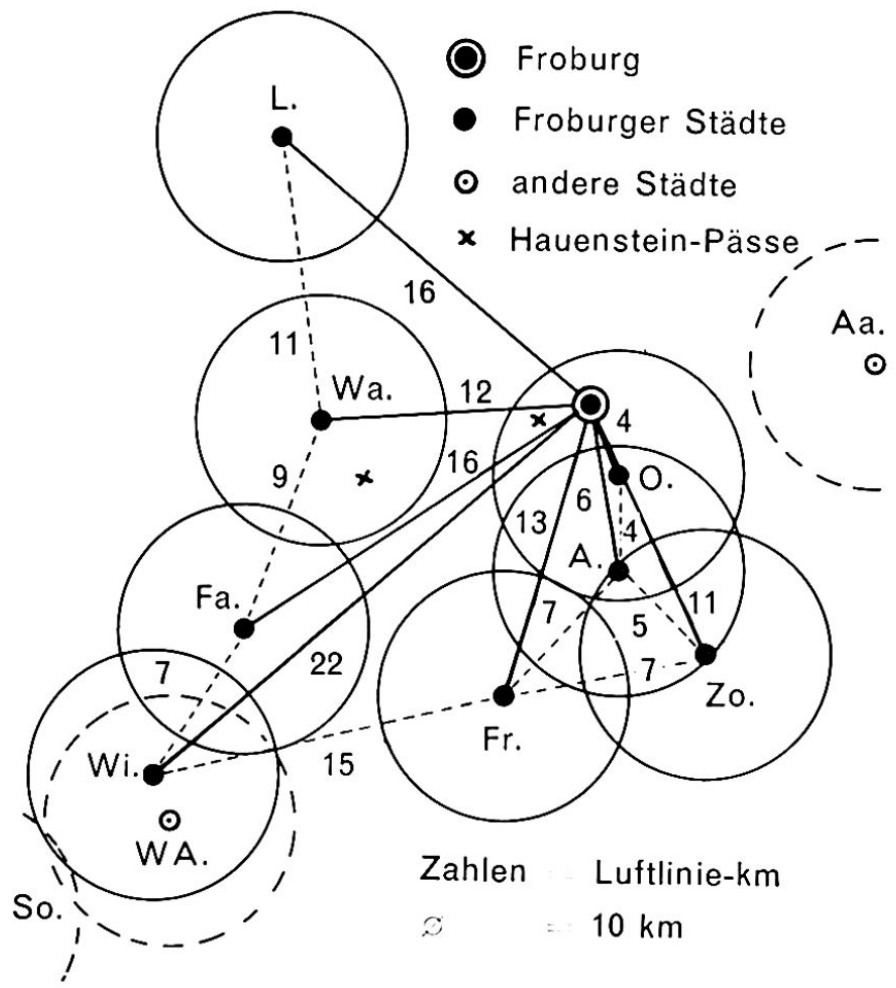

Fig. 1 Distanzen zwischen den Froburger Städten. 
Bis zum Jahre 1240 wurde sodann dieses Burgennetz durch systematische Gründung von 8 Städten verstärkt. Es sind dies von Norden nach Süden: Liestal, Waldenburg, Olten, Falkenstein, ${ }^{3}$ Aarburg, Zofingen, Fridau und Wiedlisbach.

\subsection{Die Gründungszeiten der Froburger Städte}

Gründungsurkunden fehlen für alle acht Städte, und da zudem schriftliche Dokumente aus jener Zeit sehr spärlich vorhanden sind, kann stets nur der sptätestmögliche Zeitpunkt der Gründung angegeben werden.

Zofingens Gründung reicht wahrscheinlich schon ins 12. Jahrhundert zurück, denn schon 1227 erwirbt sich das Kloster St. Urban dort ein Haus und 1230 werden erstmals "cives» genannt.

Liestal heißt 1241 «burgum», was bereits auf eine Befestigung schließen läßt.

Waldenburg führt 1244 den Namen «civitas».

Fridau ist 1263 ein "municipium» und es treten auch «burgenses» auf.

Aarburg und Wiedlisbach sind wahrscheinlich 1263 unter den froburgischen "oppida» gemeint. Ganz sicher ist aber Wiedlisbach 1275 Stadt; Aarburg hingegen heißt erst wieder 1330 "stat».

Olten hat 1263 einen Schultheißen und tritt 1265 unter «munitio» auf. Wie der Name Zofingens taucht aber auch derjenige Oltens bereits 1201 in den Urkunden auf.

Falkenstein schließlich wird 1315 neben dem Schloß «stettlin» genannt, doch schon 1255 wird ein Torwächter erwähnt (18).

\subsection{Die Entstehungsarten}

Von den acht Städten knüpft allein Olten an eine römische Siedlung (castrum) an. Doch dürfte seit der Römerzeit der Platz kaum ununterbrochen besiedelt und befestigt gewesen sein. In der Völkerwanderungszeit erfolgten bestimmt durch die Alamannen beträchtliche Zerstörungen. Sicher ist, daß im 12. Jahrhundert eine froburgische Ministerialenfamilie im Schloß Zielemp über der Aare saß. Die Stadt wurde dann von den Froburgern planmäßig angelegt, unter Einschluß von Kirche und Burg und unter teilweiser Benutzung der römischen Mauerreste. ${ }^{4}$ Der regelmäßige Grundriß der Stadt beweist, $\mathrm{da} ß$ es sich um eine Stadtgründung und nicht um ein allmähliches Wachstum handelt.

In Zofingen diente eine alamannische Siedlung als Grundlage. Der Ort war Mittelpunkt des untern

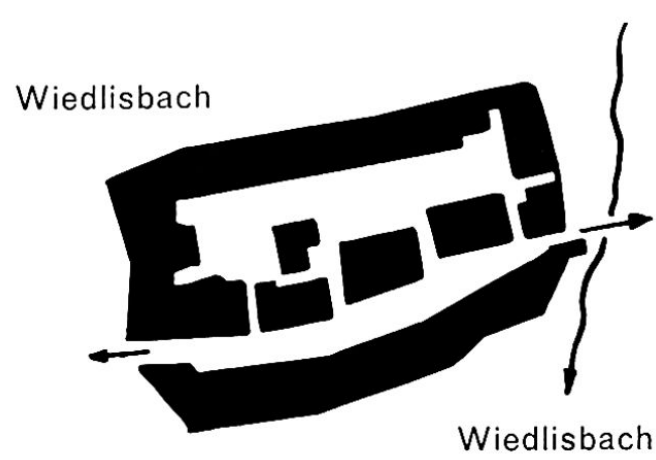

Wiedlisbach

Waldenburg

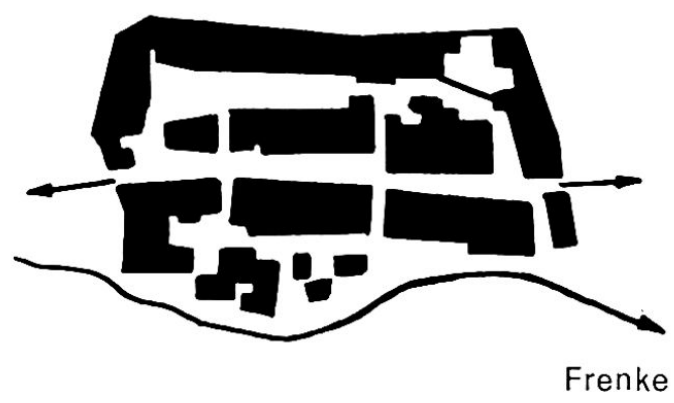

Fig. 2 Grundriß von Wiedlisbach und Waldenburg.

Wiggertales, und die Froburger besaßen hier im 12. Jahrhundert einen ansehnlichen Herrenhof. Im Verlaufe des Jahrhunderts verwandelten sie die Pfarrei in ein Chorherrenstift, das sie aus ihrem Besitz reich mit Gütern ausstatteten. Zu dem Bestand von Dorf, Herrenhof und dem kreisförmigen Stiftsbezirk fügten dann die Froburger eine ziemlich regelmäßig angelegte Marktsiedlung, die heutige Oberstadt, befestigten das Ganze und erhoben es zur Stadt. Auch hier liegt somit eine planmäßige Gründung vor.

Ähnlich war der Vorgang in Liestal. Auch da waren eine alte Kirche und eine entsprechende Siedlung vorhanden, zu denen sich ein froburgischer Herrenhof gesellte. Zum alten unregelmäßigen Siedlungsbezirk mit der Kirche wurde eine regelmäßige Marktsiedlung mit breiter Hauptgasse gefügt und mit einer Befestigung versehen. Von planmäßiger Gründung kann auch da gesprochen werden.

Waldenburg, Falkenstein, Wiedlisbach, Fridau und Aarburg knüpfen an keine frühere Siedlung von Bedeutung an. Waldenburg, Falkenstein und Aarburg entstanden unterhalb bestehender Burgen und sind wohl zu einem guten Teil zur Verstärkung der StraBen- und Talsperre geschaffen worden. Auffallend ist, daß diese fünf Städte ursprünglich keine eigene Kirchgemeinde bildeten, sondern zu einer alten Dorfpfarrei gehörten. 
Alle acht Froburger Städte sind somit gegründete Städte, bei deren Anlage nach bestimmtem, meist ähnlichem Muster vorgegangen worden war.

\subsection{Anlage und Grundri $\beta$}

Die Anlage der acht Städte zeigt, daß überall erfahrene Städtebauer am Werk gewesen sind. Ihre Namen sind uns jedoch unbekannt. Die Absichten der Gründer treten da am besten zutage, wo sie sich an nichts Bestehendes anzulehnen hatten, so in Wiedlisbach, Waldenburg und Fridau. Auffallend ist besonders die fast völlige Übereinstimmung der Anlagen von Waldenburg und Wiedlisbach. Beides sind rechteckige Marktsiedlungen mit einer breiten Haupt- oder Marktgasse und einer parallel verlaufenden Neben- oder Hintergasse.

Auch wenn der Straßenverlauf von Fridau heute unbekannt und in keiner Weise mehr sichtbar ist, darf auch da auf eine ähnliche Anlage wie in Waldenburg oder Wiedlisbach geschlossen werden. Zwar zeichnet sich im Gelände noch immer das ehemalige Stadtareal ab, und auch der Verlauf des Stadtgrabens rund um das kleine Plateau herum ist noch festzustellen. Außer den Resten einer Kieselsteinmauer im Graben auf der Ostflanke sind jedoch keine nennenswerten Funde gemacht worden. ${ }^{5}$

Aarburgs Grundriß war durch den Verlauf von Aare und Burgfelsen vorbestimmt; er zeigt die Form eines ziemlich regelmäßigen Dreiecks, das den engen Raum völlig ausnutzt. ${ }^{6}$

In Falkenstein erlaubte das Gelände auch keine Großanlage. Urbare aus dem 16. Jahrhundert bieten hingegen Anhaltspunkte dafür, daß das Städtchen sich nicht wie heute auf die beiden Häuserzeilen beidseitig der Straße beschränkte, sondern daß der damals ganz der westlichen Berglehne folgende Bach noch einer dritten Häuserreihe Platz bot. Dafür war die ursprüngliche Nord-Süd-Ausdehnung noch kürzer, als man sie nach spätern bildlichen Darstellungen annehmen möchte (18).

Selbst in Olten, wo Kirche, Schloß Zielemp und stückweise die römische Castrumsmauer zur Anpassung zwangen, liegt das bewährte Schema vor: eine leicht gebogene und geknickte Hauptgasse, leicht zur Brücke abfallend und zwei ungefähr gleich verlaufende Hintergassen.

In Zofingen mußte an das Chorherrenstift und die Kirche angeknüpft werden. Südlich davon entstand die regelmäßig angeordnete Oberstadt mit Hauptgasse und Parallelgassen, und als dieser Stadtteil zu klein wurde, wiederholte man nördlich der Kirche, in der Niederstadt, ungefähr denselben regelmäßigen Grundriß.

In Liestal wurde das dreigassige Schema nordwest-

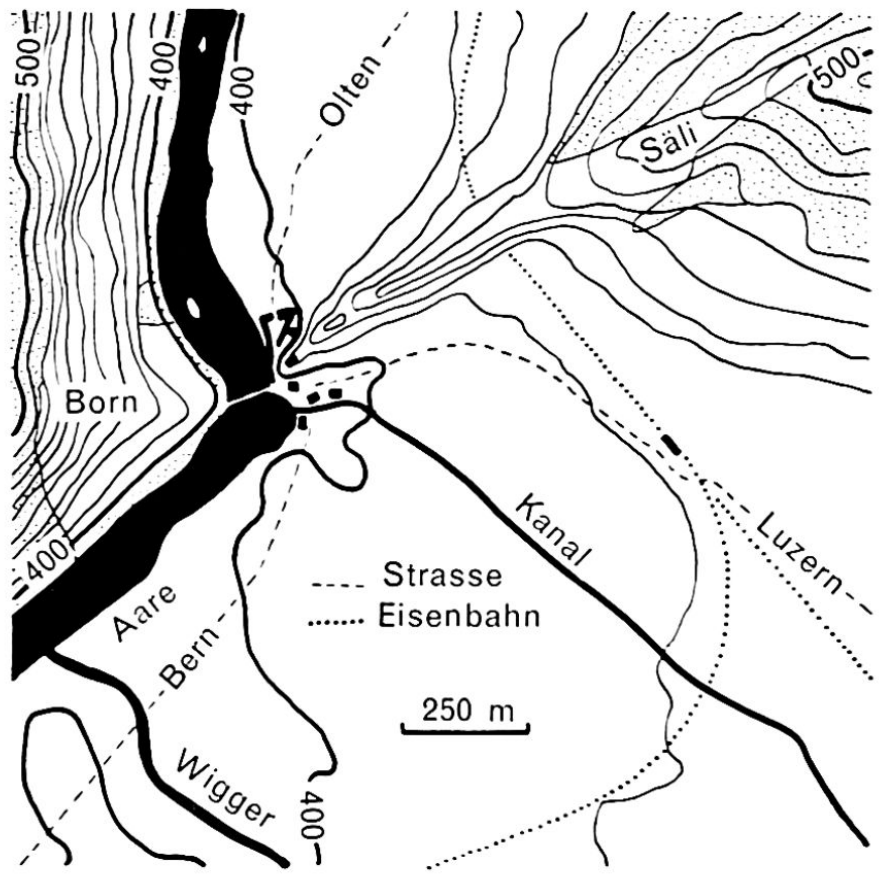

Fig. 3 Lage von Aarburg.

lich des bestehenden Bezirks um Kirche, Hof und dörfliche Siedlung angewandt.

Allen Städten gemeinsam, ob sie sich an Bestehendes anlehnen mußten oder ob sie ohne jegliche Rücksichtnahme geschaffen werden konnten, ist somit das axiale Schema. Die froburgischen Städtebauer übernahmen damit die Grundform, wie sie im mittleren und späten 12. Jahrhundert bei den zahlreichen Neugründungen im südwestdeutschen und schweizerischen Raum meistens angewandt worden ist (9b).

\subsection{Die Standorte}

Die natürliche Schutzlage, wie sie vor allem durch das Gelände gegeben wird, ist bei den acht Froburger Städten recht unterschiedlich. Äußerst ausgeprägt ist sie in Waldenburg, Aarburg, Olten, Liestal und Falkenstein, weniger offensichtlich hingegen in Fridau und Zofingen.

Eine ausgesprochene Schutzlage besitzt Olten. ${ }^{7}$ Ein schmaler Malm-Doggersporn schiebt sich auf einem Niveau von 400 Metern vom Jurahang bis ans westliche Aareufer vor, wo er senkrecht zum fast 10 Meter tiefer liegenden Fluß abbricht und diesen sichtlich um rund 20 Meter verengt. ${ }^{8}$ Eine günstigere Stelle für Brücke und Brückenkopf gab es aareauf- und aareabwärts weitherum nicht. Auch der Südrand des Sporns fällt steil ab, und genau auf der Grenze zwischen ihm und der anschließenden tiefsten Schotter- und Flußterrasse fließt die Dünnern von Westen kommend der Aare zu. Die Stadt war 
also auf zwei Seiten durch Flüsse und steile, zum Teil fast senkrechte und felsige Ufer natürlich geschützt; eines künstlichen Stadtgrabens bedurfte es nur auf der West- und Nordseite. $\mathrm{Zu}$ beachten bleibt ferner, daß auch die Umgebung des Stadtkerns zur Siedlungsanlage günstig war. Längs Aare und Dünnern sind verschiedene Terrassen festzustellen, und die südlich vorgelagerte Born-Engelberg-Antiklinale mit den froburgischen Wartburgen in der Mitte ist zudem genau südlich von Olten durch die Aarekluse Richtung Mittelland durchbrochen.

Hinter dem Südeingang dieser Klus liegt Aarburg. ${ }^{9}$ Der Südschenkel der Antiklinale (Malm) steigt hier fast senkrecht auf und bildet ein langes, schmales Band, das sich von der Wartburg (Säli-Schlößli), 663 Meter, bis zur Aare hinunterzieht, die hier rechtwinklig umbiegt und antezedent in die Falte einbricht. Auf dem westlichen Ufer des Flusses steigt dann der Schenkel erneut auf und bildet die Südseite des Bornes, 719 Meter. Die dem Mittelland zu gelegene Wiggerebene ist mit Niederterrassenschotter bedeckt, ebenso das von der Aare durchflossene Innere der diagonal verlaufenden Klus. Der Stadtkern Aarburgs, 400 Meter, liegt bereits innerhalb der Klus und direkt am Innenfuß des $467 \mathrm{Me}-$ ter hohen burgbewehrten Felsbandes. Der Durchbruch der Aare mußte offenbar noch von Menschenhand erweitert werden, damit wenigstens für die Straße Platz entstand. Für die Stadt bleibt zwischen Burgfelsen und Aare ein kleines Dreieck mit je etwa 100 Metern Seitenlänge. Der Schutz war auf der West-, Süd- und Ostseite ein vollkommener; nur im Norden, gegen Olten zu, war zusätzlicher Schutz notwendig.

Eine vollkommene Klusenlage besitzt auch Falkenstein. 10 Das Städtchen liegt direkt im nördlichen Ausgang der Dünnernkluse zwischen Balsthal und Oensingen. Der mit Schotter aufgefüllte Durchbruch im nördlichen Malmschenkel der Weißensteinkette läßt außer dem Fluß nur noch der Straße und parallel dazu verlaufenden Häuserreihen Platz. Senkrecht über dem 480 Meter hoch gelegenen Ort erhebt sich auf 520 Metern auf den östlich wieder ansteigenden Flühen die Burg Alt-Falkenstein. Lediglich zwei Talsperren von je etwa 50 Metern Breite mußten in Falkenstein angelegt werden.

In Liestal, 320 Meter, erstreckt sich von Seltisberg her bis an den südlichen Stadtrand ein gleichmäßig abfallender Dogger-Höhenzug. ${ }^{11}$ In und bei Liestal vereinigen sich Ergolz, Frenke und Orisbach, so $\mathrm{da} ß$ die geographische Lage sehr ausgeprägt ist. Die Froburger Stadt entstand da, wo der verschleppte unterste Abschnitt des Orisbaches zusammen mit der bereits durch die Frenke verstärkten Ergolz einen Riedel aus dem Schotter modelliert hat. Die Stadtmauer folgte größtenteils der Riedeloberkante, was einer ausgesprochenen Schutzlage gleichkommt. Hier streben die beiden Hauensteine auseinander,

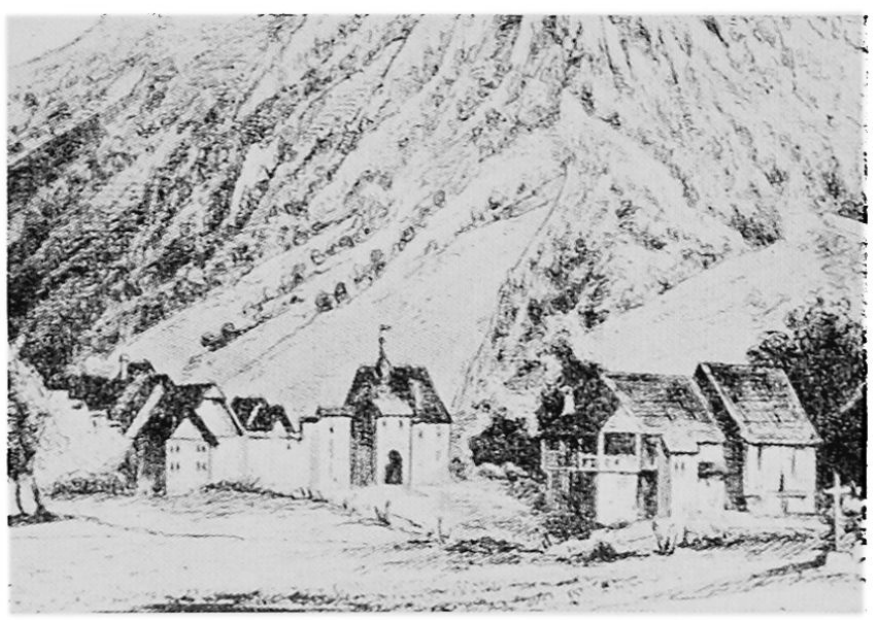

Fig. 4 Falkenstein/Klus im 18. Jahrhundert. Blick gegen Süden und die Weißensteinkette.

und die Wasserkraft ist beträchtlich; weitere für die Anlage der Stadt wesentliche Standortsfaktoren.

Waldenburg, 530 Meter, liegt im kaum mehr als 100 Meter breiten Engpaß eines Rippendurchbruches der Vorderen Frenke, der aus Hauptrogenstein besteht und von der Richtiflue, 799 Meter, im Westen nach der Gerstelflue, 929 Meter, im Osten zieht.12 Etwa $1 \mathrm{~km}$ talabwärts schließt sich bei Oberdorf die Zone des Keupers und Muschelkalks an, die dort einen Gewölbekern bilden. Außer für die Frenke und die zwei Gassen des Städtchens bleibt im Engpaß kein Raum mehr übrig. Die Lage hat eine große Ähnlichkeit mit derjenigen Falkensteins, denn auch in Waldenburg thront hoch über den Gassen das Schloß.

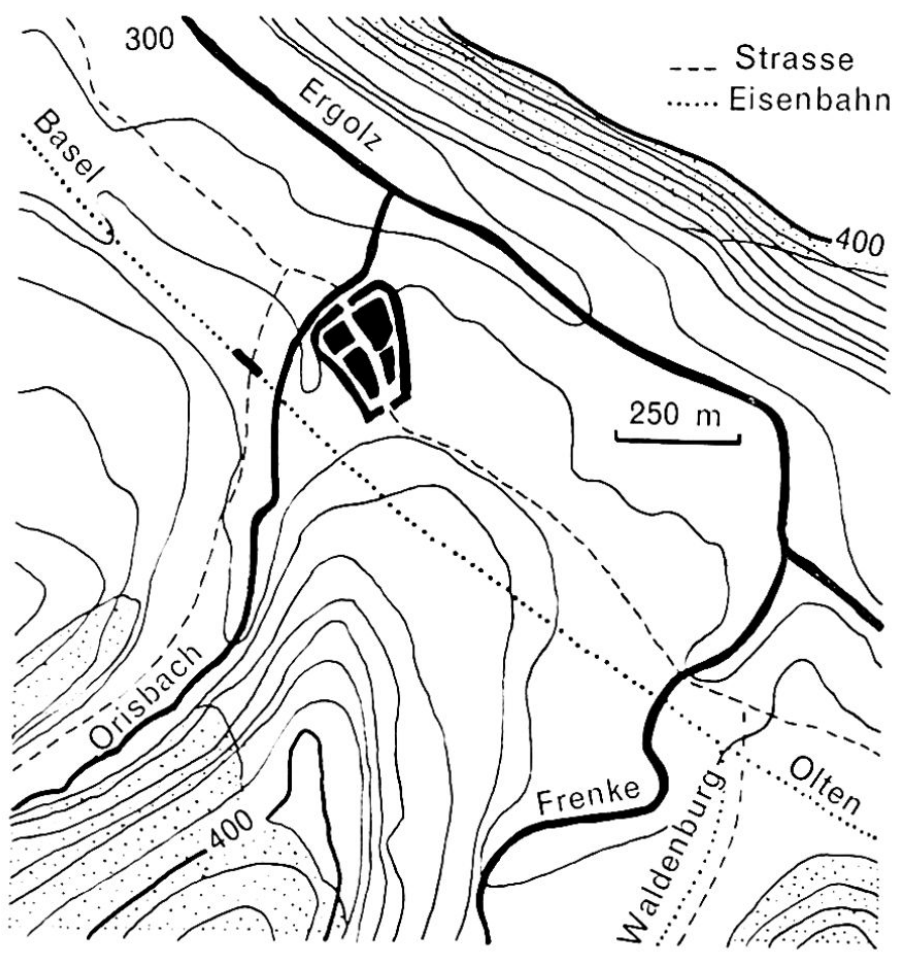

Fig. 5 Lage von Liestal. 
Wenig ausgeprägt ist die Schutzlage von Wiedlisbach. ${ }^{13}$ Der Ort, 465 Meter, liegt auf der Grundmoräne des Würm-I-Gletschers, die ihrerseits wieder auf Bergsturzmaterial der Weißensteinkette abgelagert worden ist. Die Hügel der Endmoräne erheben sich bogenförmig einige hundert Meter östlich und sind für die Stadtanlage ziemlich bedeutungslos. Bis zum Südrand der Jurakette 300 Meter nördlich Wiedlisbach steigt das Gelände nur um 20 Meter an, und gegen Süden bis zum rund 20 Meter hohen Terrassenrand über der alluvialen Aareebene fehlen bei nur geringer Neigung 600 Meter. Der Wiedlisbach, vom Jura und von Rumisberg kommend, fließt direkt am östlichen Stadtrand vorbei der Aare zu. Er bietet jedoch nur unvollkommenen Schutz und kann kaum als natürlicher Stadtgraben angesehen werden. Wiedlisbach liegt somit nach allen Seiten frei, und nicht einmal eine Moränenerhebung ist an der Stadtstelle festzustellen. Allein von der Geländebeschaffenheit aus läßt sich der Standort des Städtchens nicht erklären.

Fridaus Lage ist durch die Aare bestimmt. ${ }^{14}$ An ihrem Nordufer wird ein schmaler Streifen der Untern Süßwassermolasse angeschnitten. Das ehemalige Stadtareal unmittelbar darüber liegt 420 Meter hoch ganz auf dem Niederterrassenschotter. Zu beachten bleibt noch, daß direkt am westlichen Stadtrand ein ehemaliger Aarelauf in das heutige Bett einmündet. Diese tiefste alluviale Talsohle könnte für die Anlage Fridaus von einiger Bedeutung gewesen sein, denn die Eintiefung ist im Gelände vom westlichen Stadtrand an bis oberhalb Wolfwil noch gut feststellbar und bietet gegen Westen einen gewissen Schutz.

Nicht im geringsten geschützt ist das froburgische Zofingen. ${ }^{15}$ Es liegt zu allen Teilen auf dem fast topfebenen Talgrund der Niederterrasse am Ostrand des Wiggertales, rund 430 Meter hoch. Der

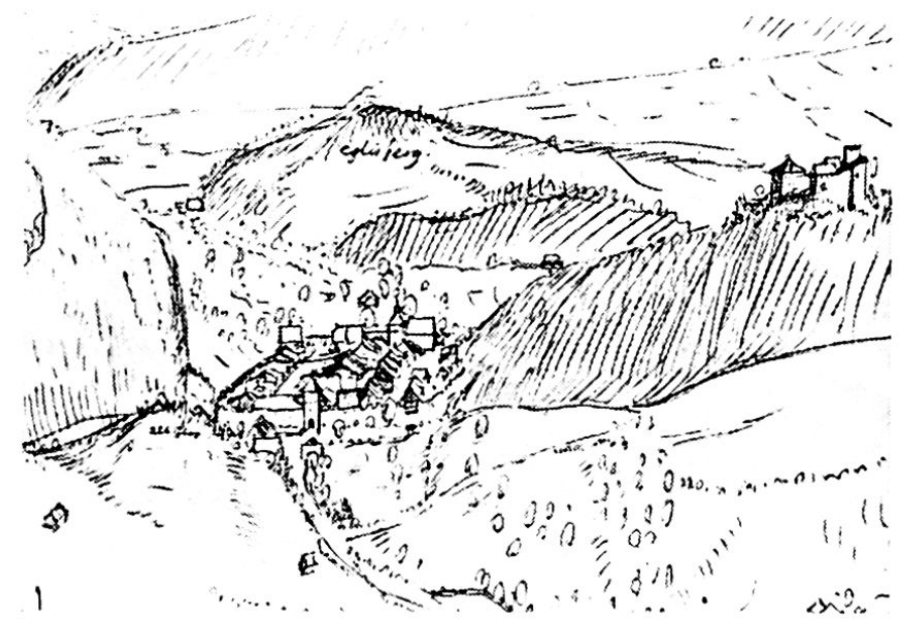

Fig. 6 Waldenburg, Blick talabwärts. Ausschnitt aus einer Feldaufnahme G.F.Meyers, 1645-1693. Hrsg. 1960 vom Baselbieter Heimatschutz.

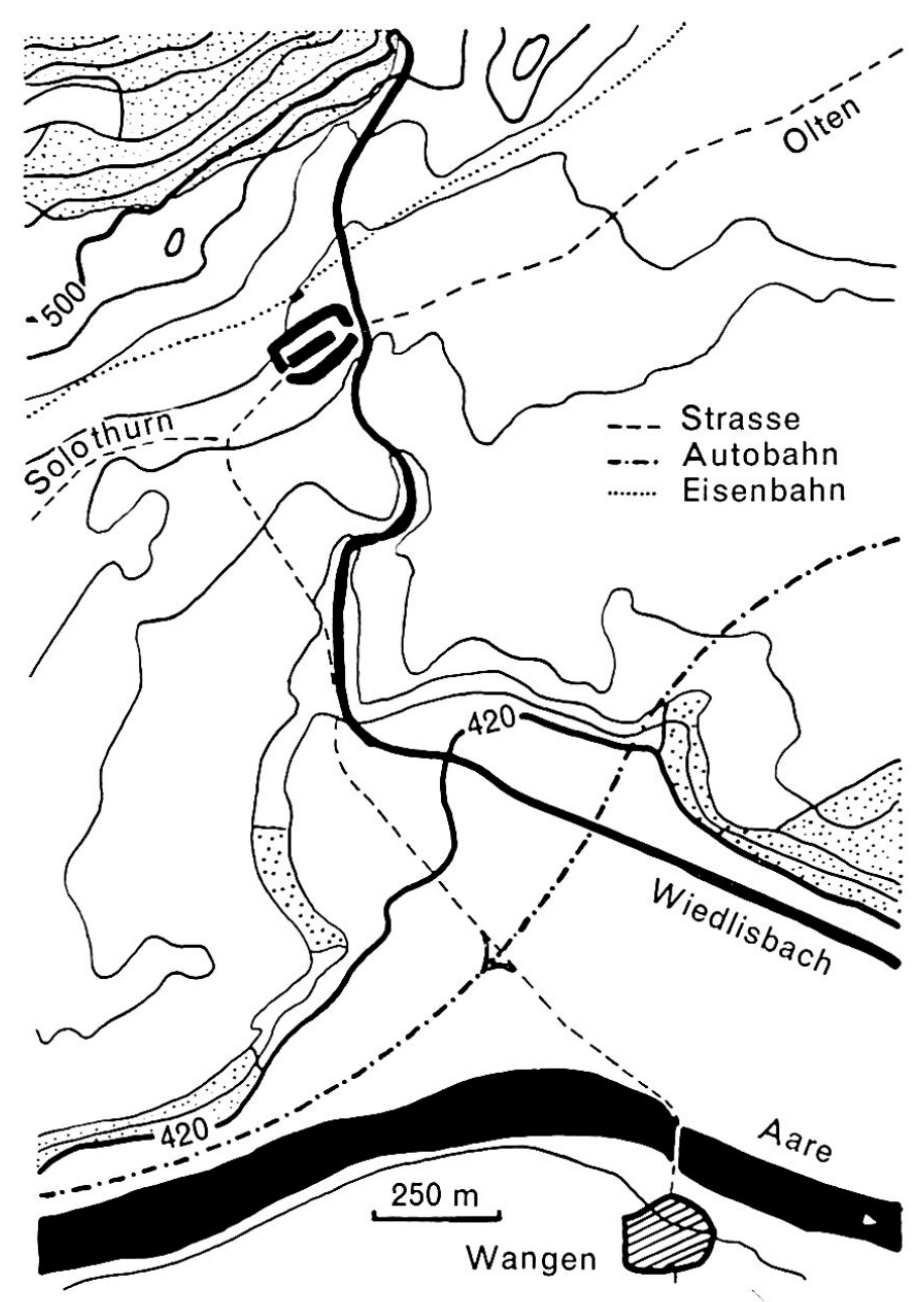

Fig. 7 Lage von Wiedlisbach.

Fluß bietet keinerlei Schutz, fließt er doch fast 1 Kilometer westlich der Stadt vorbei. Erst nach dem ostseitigen Stadtgraben beginnt das Gelände allmählich über einige diluviale Schuttkegel zur Hochterrasse und dem daran anschließenden Tertiär leicht anzusteigen. Hier haben kleine Seitenbäche ein paar die Stadt überblickende Anhöhen herausmodelliert, die um 500 Meter hoch liegen. Gegen Süden, Westen und Norden liegt Zofingen völlig offen.

Unterschiedlich ist auch die Fernverkehrslage der acht Städte, doch scheint es, daß die Froburger darauf noch mehr Gewicht gelegt haben als auf eine gute Schutzlage. Mit Ausnahme Fridaus liegen alle an Paßstraßen oder deren Zufahrten: Waldenburg, Falkenstein und Wiedlisbach am Obern, Olten, Aarburg und Zofingen am Untern Hauenstein; Liestal befindet sich genau an der Gabelung der beiden Übergänge. Vor Fridau zog der Verkehr durch das Aaretal, und die Stadt war mit ihrem Flußübergang ein Verbindungsglied an der direkten Route von Zofingen nach Wiedlisbach.

Etwas überraschend sind höchstens die Verkehrslagen von Zofingen und Wiedlisbach. Warum ist Zo- 
fingen nicht $3 \mathrm{~km}$ weiter nördlich beim heutigen Oftringen an der Kreuzung der Hauenstein-Gotthardstraße mit der großen West-Ost-Route entstanden? Oder warum entstand $3 \mathrm{~km}$ weiter südlich beim froburgischen Sitz Wikon keine Stadt, wo doch die Südgrenze des froburgischen Besitzes verlief? Offenbar ließ man sich durch die bereits bestehende Siedlung zum Standort verleiten. Jenseits der Aare, nur $2 \mathrm{~km}$ von Wiedlisbach entfernt, lag das nichtfroburgische Wangen a. A. Warum entstand Wiedlisbach nicht an der Westgrenze des Buchsgaus, $3 \mathrm{~km}$ weiter westwärts an der Siggern? $\mathrm{Da}$ Wiedlisbach nicht vor dem südlichen Kluseingang beim heutigen Oensingen entstand, ist höchstens so zu erklären, daß jene Gegend im Einflußbereich der Bechburger lag.

\subsection{Ausdehnung und Einwohnerzahl}

Für die Zeiten nach der Gründung im Hochmittelalter finden sich in Lit. 1 die folgenden Angaben:

$\begin{array}{cl}\text { ungefähre } & \text { ungefähre } \\ \text { Fläche, ha } & \text { Einwohnerzah } \\ 8 & 1200-1500 \\ 4,5 & \text { unter } 1000 \\ 1,5 & \text { um 200 } \\ 1,5 & \text { um 200 } \\ \text { um } 1,5 & ? \\ 1,3 & \text { um 200 } \\ 0,5 & \text { unter 200 } \\ 0,5 & \text { unter } 200\end{array}$

Vergleichsweise umfaßte Solothurn in derselben Zeit rund 12 ha; die größten Städte des Mittelalters überdeckten um die 100 ha. Basel als die damals größte Schweizer Stadt zählte um 10000 Einwohner und die Mittelstädte bewegten sich etwa zwischen 2000 und 5000. Alle froburgischen Gründungen waren somit ausgesprochene Kleinstädte.

\subsection{Struktur und Funktionen}

Sechs Aspekte des wirtschaftlichen und sozialen Aufbaus, der Verkehrslage, der rechtlichen, politischen und architektonischen Gestalt sind es, aus deren Überschneidung das Bild der mittelalterlichen Stadt entsteht: die Wirtschaftsstruktur (Markt, Gewerbe, Handel), die Verkehrslage (Straßen, Brücke, Rastort, Umschlagplatz), die Rechtspersönlichkeit (Kommunalverfassung, Organe, Rechtskreis), die bauliche Gestalt (Stadtplan, Gassennetz, öffentliche Gebäude, Stadtbefestigung), die politi-

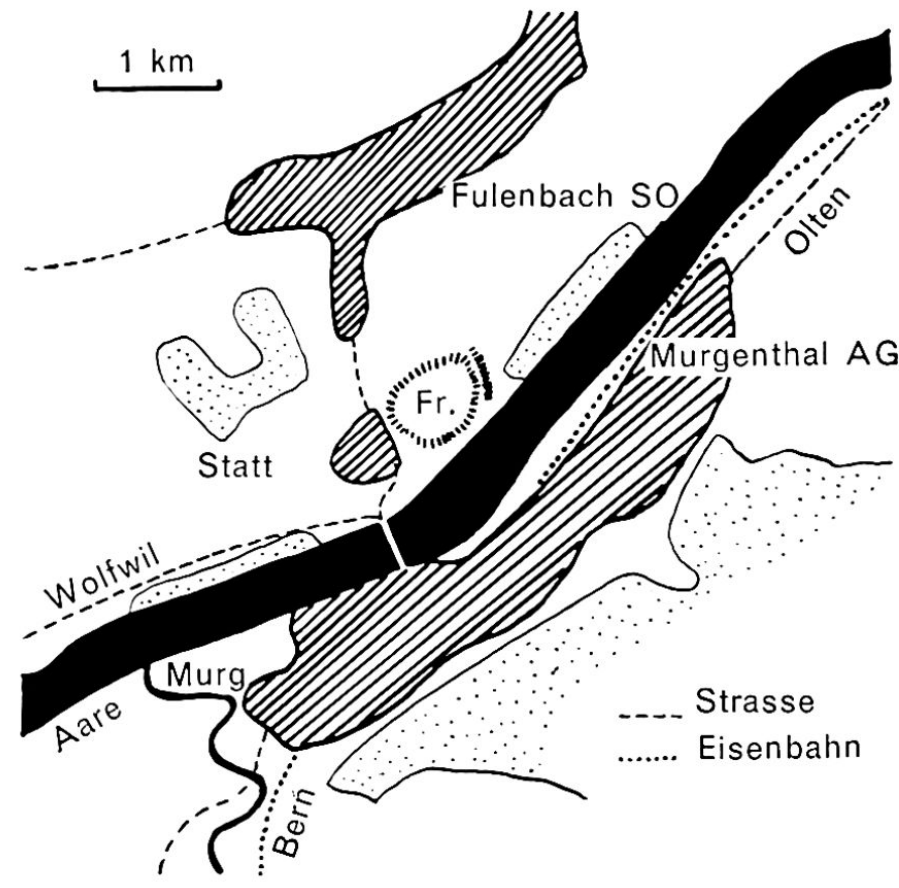

Fig. 8 Lage von Fridau.

sche Vitalität (Subjektcharakter) und die Sozialstruktur (Handwerker, Kaufleute, Geistlichkeit, Patriziat). Jede einzelne der sechs Wertgruppen kann nur in Ansätzen vorhanden sein oder ganz verkümmern; wo sie vollständig da sind und sich gleichmäßig entwickeln, erscheint das Bild einer vitalen, kräftig ausgreifenden Stadt, bei lediglich zwei oder drei Wertgruppen bleibt die Gemeinde, wenn überhaupt fortlebend, Zwergstadt (9b).

Wie allein schon die Einwohner- und Flächenzahlen gezeigt haben, trifft die letzte Feststellung weitgehend auf die mittelalterlichen Froburger Städte zu. Wenn man diese mit dem sechsseitigen Diagramm in Lit. $9 \mathrm{~b}$ in Verbindung bringt, wird diese Tatsache bestätigt. Unser diesbezüglicher Versuch muß allerdings in einigen Teilen fragwürdig bleiben, da nicht für alle acht Städte die hierfür notwendigen rechts- und wirtschaftshistorischen Untersuchungen vorliegen oder überhaupt die urkundlichen Berichte fehlen. ${ }^{16}$

Gesamthaft gesehen war somit eine Hauptfunktion aller froburgischen Städte, anstelle oder zusammen mit den vielen Burgen den Verkehr von Basel her über den Jura Richtung Alpen zu beherrschen und das Gebiet der Froburger militärisch zu sichern. In keiner Stadt hatte das Gewerbe ausgesprochen überregionale Bedeutung. Nirgends gab es nennenswerten Export und nirgends wurde etwas Besonderes erzeugt; das gewerbliche Leben bewegte sich überall in recht engen Bahnen. Den stärksten Handel fand man zweifellos in Zofingen. Urkundlich sind einige Zofinger auswärts belegt, und es ist kein Zufall, daß hier schon früh im 13. Jahrhundert die Froburger ihre Münzstätte aufschlugen. Die Landwirt- 
am stärksten in Zofingen und Liestal

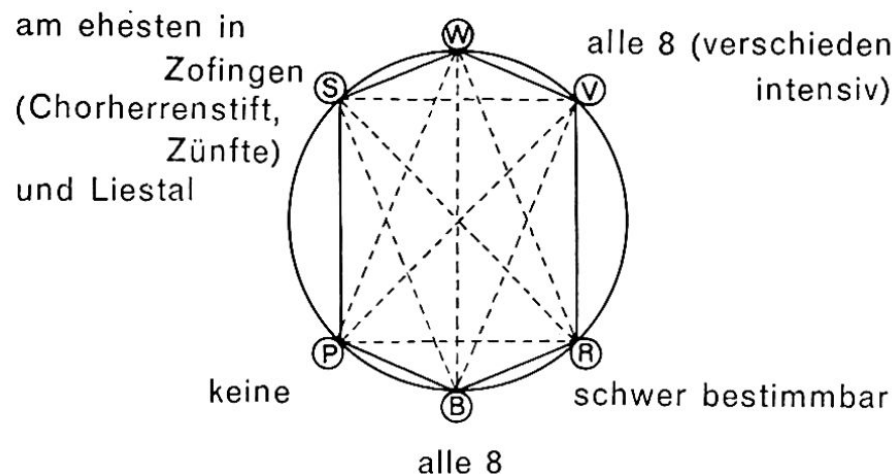

W: Wirtschaftsstruktur, V: Verkehrslage,

R: Rechtspersönlichkeit, B: Bauliche Gestalt,

P: Politische Vitalität, S: Sozialstruktur

Fig. 9

schaft spielte noch überall eine Rolle. Auch Zofingen besaß unter seinen Zünften eine besondere Ackerleutegesellschaft. Das bäuerliche Element war sicher in den andern, kleinern Städten noch stärker, wenn auch nirgends überwiegend. Am wenigsten nahmen die Froburger Rücksicht auf die Marktlage. Waldenburg besaß einzig ein langgezogenes, enges Tal als Einzugsgebiet, Wiedlisbach lag zu nahe bei Wangen a. A. und Solothurn, Liestal spürte die Nähe Basels und Olten, Aarburg, Zofingen und Fridau lagen überhaupt viel zu nahe beieinander, so daß sie sich gegenseitig konkurrenzierten. Figur 1 zeigt die erstaunlich geringen Distanzen von der Stammburg und von Stadt zu Stadt. Von den froburgischen Wartburgen aus waren zum Beispiel die vier Städte Olten, Aarburg, Zofingen und Fridau direkt zu überblicken. Im Mittelalter gehörte diese Region zu der Zone mit der höchsten Städtedichte überhaupt (9b).

Hatten die Froburger die Marktlage und überhaupt das Wirtschaftliche falsch berechnet und eingeschätzt? Möglich wäre, daß das Einzugsgebiet für die Froburger nur von zweitrangiger Bedeutung war und außer militärischen auch politische Aspekte mitgespielt haben. Von Historikern wird heute angeführt, daß die Grafen von Froburg dem Burgenbau des niederen Adels längs der HauensteinstraBen feindlich gesinnt gewesen seien und mit den Städtegründungen ein Gegengewicht dazu schaffen wollten. Jedenfalls ist die Tatsache, daß auf dem verfïgbaren Raum zu viele Städte angelegt worden sind und daß dadurch die unbedingt notwendigen wirtschaftlichen Einzugsgebiete zu klein ausfallen mußten, am stärksten dafür verantwortlich, daß bis ins 19. Jahrhundert keine der acht Städte sich auch nur einigermaßen entwickeln konnte, mit der jüngsten wirtschaftlichen Entfaltung im 20. Jahrhundert aber im Gebiet von Olten-Aarburg-Zofingen eine Siedlungsagglomeration mit all den dazu gehörenden, schwer lösbaren Problemen entstanden ist.

\section{Die geschichtliche Entwicklung bis zum 19. Jahrhundert}

Keiner der acht Froburger Städte ist es gelungen, das Aussterben des Grafengeschlechtes zur Erlangung der Unabhängigkeit oder Reichsunmittelbarkeit auszunützen. Alle wechselten lediglich die Herrschaft und wurden mehr oder weniger politische Objekte. Verhängnisvoll wirkte sich vor allem der Guglereinfall von 1375 aus. Vom Elsaß her waren die beiden Hauensteine die Anmarschwege ins Mittelland: Waldenburg, Wiedlisbach, Falkenstein und Fridau erlagen, und nur Liestal und Olten vermochten standzuhalten. Während Waldenburg und Wiedlisbach im alten Umfang wieder aufgebaut wurden, verschmolz Falkenstein später mit der Gemeinde Balsthal, von der es heute eine Art Industrievorort (von Roll'sche Eisenwerke) ist. Ein gewisses Eigenleben innerhalb der Gemeinschaft war aber seit je vorhanden, bildete doch Klus eine eigene Bürgergemeinde, die 1484 erstmals erwähnt wird. Seit 1440 die Glasmacherei und ab 1480 die Eisenverarbeitung gaben dem Ort zudem stets ein eigenes Gepräge. Fridau verschwand sogar völlig, und heute ist auf dem Gebiet der solothurnischen Gemeinde Fulenbach lediglich noch der Flurname «Statt» vorhanden, der sich auf eine Häusergruppe nördlich der Aarebrücke Fulenbach-Murgenthal bezieht. Fridau und Falkenstein können somit in den folgenden Betrachtungen weggelassen werden.

Auch räumlich war die Entwicklung der übrigen sechs Städte unbedeutend. Einzig Zofingen bekam noch am Ende der Froburgerzeit, spätestens jedoch 1285, eine Unterstadt. Eine kleine Vorstadt außerhalb der Mauern ist zunächst nur in Aarburg um Mühle und Säge auf der Südseite des Burgfelsens anzutreffen.

Die politische Entwicklung der sechs überlebenden Städte nahm, kurz zusammengefaßt, folgenden Verlauf:

Nach dem Aussterben der Froburger fiel Olten an den Bischof von Basel zurück. Es folgten verschiedene Verpfändungen, 1426 diejenige an Solothurn, das es 1532 als Eigentum erwarb. Olten verlor mit den Jahren fast alle seine früheren Rechte, so daß sein letzter Stadtbrief von 1795 nicht viel besser aussah als ein üblicher Dorfbrief. Größere politi- 
sche Aktivität trat im solothurnischen Untertanenstädtchen erst wieder mit dem Franzoseneinfall von 1798 auf, und 1830 stand die dann siegreiche demokratische Bewegung im Kanton unter Oltens Führung.

Zofingen, ehemals größte Froburgerstadt, kam 1299 unter die Herrschaft der Habsburger, die der Stadt jedoch viele Freiheiten gewährten. 1415, im Zusammenhang mit der Eroberung des Aargaus, wurde Zofingen bernisch. Die Berner ließen dem Ort die meisten habsburgischen Freiheiten, so daß er, wie vor allem der Marktrodel von 1590 zeigt, seit der Reformationszeit relativ erfreulich gedieh, gleichzeitig aber auch Bern in etlichen Kriegszügen Tribut zahlen mußte. Die Helvetik brachte sodann die Loslösung von Bern: Zofingen wurde aargauischer Bezirkshauptort.

Liestal kam zunächst an die Grafen von Homburg und 1305 durch Verkauf an den Bischof von Basel. Im großen Erdbeben von 1356 wurde die Stadt stark zerstört und 1373 an Österreich verpfändet. Im Jahre 1400 wurde sie an die Stadt Basel verkauft, mit der sie 1501 eidgenössisch wurde. Basel schränkte im Bauernkrieg die Rechte Liestals beträchtlich ein, jedoch 1798 erhob sich hier der erste Freiheitsbaum der deutschen Schweiz. 1832, mit der Trennung des Kantons Basel, wurde Liestal Hauptstadt des Halbkantons Baselland.

Waldenburg gelangte vorerst ebenfalls an das Bistum Basel, das es 1400 an die Stadt verpfändete. Dieser wurde es 1585 endgültig abgetreten, als Entschädigung für dem Bischof geleistete Dienste.

Anfangs des 14. Jahrhunderts kam Wiedlisbach an die Grafen von Neuenburg-Nidau, und 1387 wurde es Ingelram von Coucy zugesprochen. Nach dem Aussterben der Neuenburg-Nidauer Grafen gelangte es an die Thiersteiner, die Wiedlisbach an Kiburg verkauften. Später war es im Besitz von Leopold III. von Österreich, der es 1405 an Egon von Kiburg verpfändete. Dann erfolgte die Abtretung an Bern und Solothurn. Ab 1463 war die Stadt Bern alleinige Besitzerin.

Auch Aarburg ging Ende des 13. Jahrhunderts an Habsburg über und kam somit 1415 ebenfalls in bernischen Besitz. Im 17. Jahrhundert bauten die Berner die Burg zur mächtigen Festung aus, denn ihre strategische Lage im schmalen Verbindungsstreifen zwischen den evangelischen Orten Bern und Zürich war offensichtlich. Die bernische $\mathrm{Fe}$ stung hat übrigens zur selben Zeit auch Festungspläne für das katholisch-solothurnische Olten zur Folge gehabt, die jedoch nie ausgeführt worden sind. Schon früher entstanden in Aarburg am suidlichen Festungsfuß längs eines Kanals, der von der Wigger abgeleitet worden war, verschiedene Gewerbebetriebe. Zudem war Aarburg ein wichtiger AareUmschlaghafen, dessen Anlage durch besondere Strömungsverhältnisse begünstigt wurde.

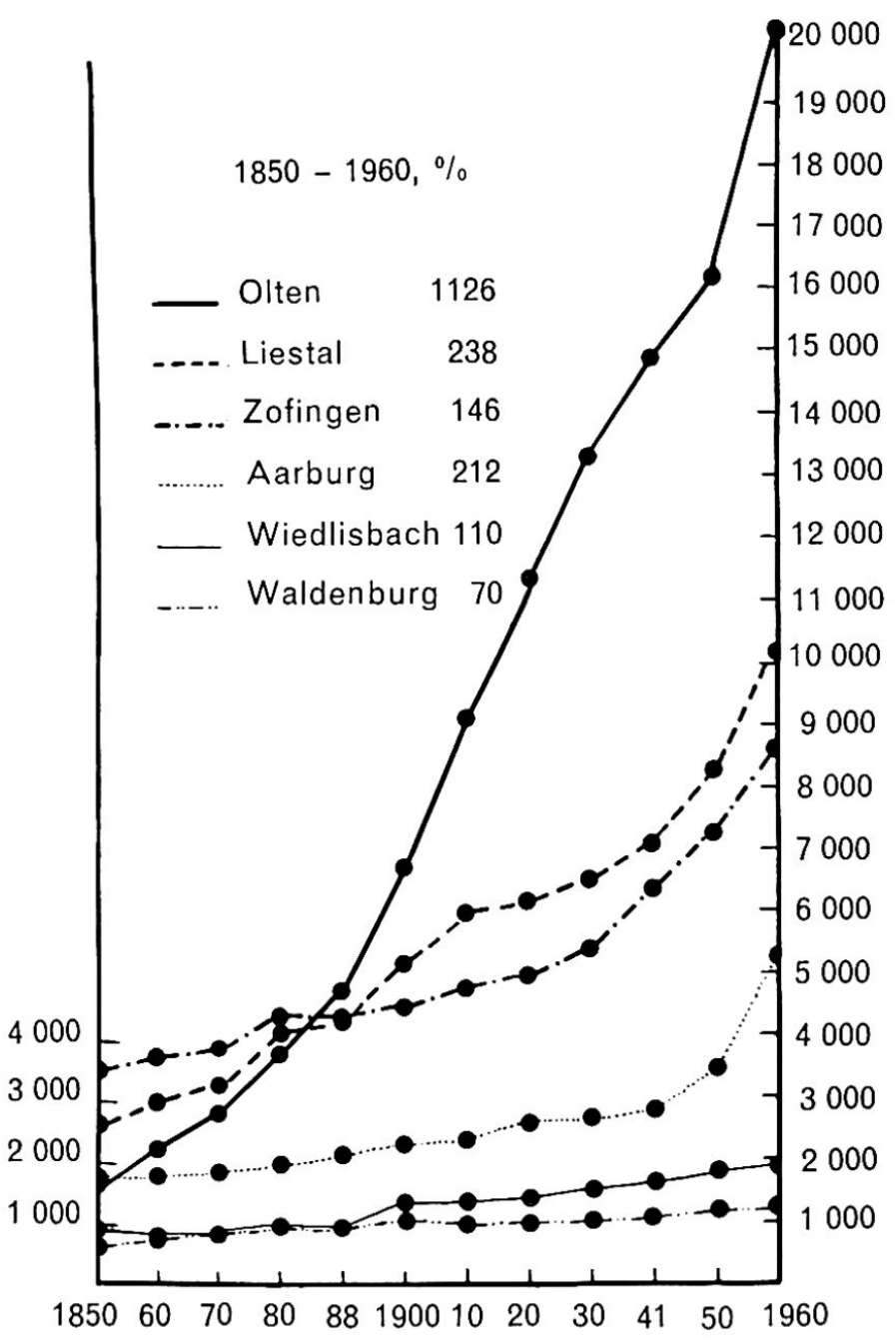

Fig. 10 Bevölkerungsbewegung 1850-1960.

\section{Die Froburger Städte heute}

\subsection{Bevölkerungsbewegung}

Figur 10 zeigt, daß die sechs Städte 1850 nach Einwohnerzahlen noch nahe beieinanderlagen, in einer Reihenfolge, die von der mittelalterlichen nur unwesentlich abweicht und deutlicher Ausdruck der politisch-wirtschaftlichen Entwicklung bis ins 19. Jahrhundert ist. Wenig mehr als 100 Jahre später jedoch, 1960, liegen die Kurven weit auseinander; Olten steht mit Abstand an der Spitze.

Wie bereits erwähnt, litten alle Froburger Städte unter ihrer politischen Abhängigkeit und vor allem unter zu kleinen, einander sich erst noch konkurrenzierenden Einzugsgebieten. Wenn sich also eine Stadt in neuerer Zeit gleichwohl aus dem bisherigen Eingeengtsein und der bescheidenen Stellung lösen konnte, war dies nur dank einer neu hinzugekommenen, in den andern fünf Städten nicht oder noch nicht auf gleicher Stufe vorhandenen Siedlungsfunktion möglich. Dies ist vor allem in Olten, das nach 1856 zum schweizerischen Eisenbahnknoten- 
punkt wurde,dann aber auch in Liestal, welches 1832 die Funktion einer Kantonshauptstadt bekam, der Fall. Diese neuen, in den andern vier Städten nicht vorhandenen Funktionen zogen ihrerseits wieder weitere an oder verschafften gegenüber den übrigen Städten vor allem einen Vorsprung in der Industrialisierung.

Olten stand 1850 in Bezug auf die Einwohnerzahl erst an vierter Stelle, und selbst die Nachbarstadt Aarburg und sogar einige Dörfer der Umgebung waren größer. Mit der Eröffnung des Bahnhofes und der Werkstätten der damaligen Centralbahngesellschaft setzte aber ein Aufschwung ein, welcher der ausgezeichneten Verkehrslage angemessen war und bis heute nicht mehr aufgehört hat (Einwohnerzahl 1966: rund 23 000). Es ist festgestellt worden, daß in der Folgezeit die meisten Industriebetriebe in erster Linie wegen der günstigen Eisenbahnverbindungen den Standort Olten gewählt haben $(8,24)$. Die ganze Wirtschafts- und Bevölkerungsstruktur ist dadurch in für schweizerische Verhältnisse recht kurzer Zeit grundlegend verändert und auch die gesamte Siedlungsanlage durch die Bahn weitgehend umgestaltet worden.

Liestal ist seit 1832 Kantonshauptort. Diese hohe Funktion hat ebenfalls schon früh andere Funktionen angezogen, wobei in Liestal in diesem Zusammenhang sicher zuerst diejenigen des Kantonsspitals und des Waffenplatzes zu erwähnen sind. So ist es nicht verwunderlich, daß seither auch die Liestaler Bevölkerungskurve recht steil ansteigt und nur zwischen 1910 und 1930 eine gewisse Verflachung aufweist, ein Umstand übrigens, der zur gleichen Zeit in Olten in keiner Weise vorhanden ist und somit auf die ganz verschiedenartige heutige Struktur der beiden ehemaligen Froburger Städte hinweist.

Bei Aarburg fällt auf, daß die Einwohnerzahl von 1850 bis 1941 nur unwesentlich angestiegen ist, dann aber auf einmal stark zunimmt. Diese Tatsache ist ziemlich eng mit der Entwicklung des nahen Olten verknüpft. Olten hat seit einiger Zeit keine größeren Industrieareale mehr zur Verfügung und auch das Wohnbauland wird zunehmend rarer. Es ist deshalb anzunehmen, daß etliche neuere Industrien in Olten keine günstigen Areale mehr gefunden und sich folglich im nahen Aarburg niedergelassen haben, dessen bauliche Entwicklung immer mehr in die Klus hinein bis an die Stadtgrenze Oltens sich verschiebt. Es entstanden hier Quartiere und Industrien, die vom Kern Oltens kaum weiter entfernt liegen als von demjenigen Aarburgs.

Zofingens Einwohnerkurve nimmt einen ähnlichen Verlauf: eine starke Zunahme setzte hier ebenfalls spät, etwa 1930, ein. Begründen läßt sich diese Tatsache zu einem guten Teil ebenfalls mit der Raumknappheit im Verkehrszentrum Olten. Die ganze Region Zofingen hat vor allem in den letzten
Jahren eine überaus intensive Industrialisierung erfahren, was gleichzeitig die Wohnzonen rasch anwachsen ließ.

Relativ wenig hat sich in den letzten 110 Jahren Wiedlisbach vergrößert. Das Bipperamt besitzt dem Jurafuß entlang noch verhältnismäßig wenig Industrien, was teilweise mit der Linienführung der SBB zusammenhängen dürfte. Nur durch Aare und neuerdings durch die Autobahn getrennt, schließt sich südwärts an der Bahnlinie Olten-Solothurn-Biel Wangen a. A. an, das zum Beispiel mit dem Waffenplatz bedeutend höhere Funktionen innehat. Auch Solothurn liegt zu nahe, und schließlich bleibt zu beachten, daß auf drei Seiten und in naher Distanz von Wiedlisbach die Kantonsgrenze verläuft. Die geringste Zunahme hat Waldenburg zu verzeichnen. Bei Betrachtung seiner Klusenlage ist dies nicht weiter verwunderlich. Zunächst fehlt hier ganz einfach das notwendige, einigermaßen ebene und weiträumige Areal - die Schmalspurbahn von Liestal her konnte nicht einmal ganz bis zur Stadt herangeführt werden - und außerdem besitzt das Städtchen überhaupt kein Einzugsgebiet. Es ist bezeichnend, daß da, wo das Vordere Frenketal etwas breiter und offener wird als in Waldenburg, sich Gemeinden befinden, die in den letzten Jahren relativ rascher zugenommen haben oder heute sogar größer sind als Waldenburg. ${ }^{17}$ Ferner ist zu berücksichtigen, daß seit 1858 mit der Eröffnung der Bahn durch den Untern Hauenstein der Paßverkehr über den Obern Hauenstein stark zurückgegangen und erst wieder im Automobilzeitalter neu erstanden ist.

\subsection{Berufs- und Wirtschaftsstruktur}

$$
\begin{gathered}
\text { Erwerbsgruppen } \\
\text { Primär \% Sekundär \% Tertiär \% }
\end{gathered}
$$

$\begin{array}{llll}\text { Olten } & 0,8 & 52 & 47 \\ \text { Liestal } & 3 & 52 & 45 \\ \text { Zofingen } & 3 & 68 & 29 \\ \text { Aarburg } & 2 & 70 & 28 \\ \text { Wiedlisbach } & 9 & 60 & 31 \\ \text { Waldenburg } & 6 & 70 & 23\end{array}$

Olten und Liestal bilden bezeichnenderweise eine Gruppe für sich. Beide haben die geringsten Anteile an den Industrieberufen, dafür die größten bei den Dienstleistungen. Olten beherbergt wegen des $\mathrm{Ei}$ senbahnknotenpunktes und der zentralen Lage auch im Straßenverkehr überdurchschnittlich viele SBBAngestellte und Angehörige anderer Verkehrsberufe (24); in Liestal machen sich unter anderem die Funktionen der kantonalen Verwaltung und des Waffenplatzes bemerkbar. In einer Darstellung mittels Dreieckkoordinaten würden sich beide Städte 
unmittelbar an der Grenze zu den gemischtberuflichen Gemeinden befinden.

Zofingen und Aarburg gleichen sich in der Struktur ebenfalls. Beide müssen als ausgesprochene Industriestädte bezeichnet werden, deren Nachbarorte ebenfalls reichlich mit Fabriken versehen sind. In Zofingen ist unter anderem die graphische und chemische Industrie vertreten; in Aarburg ist seit langer Zeit die Textilbranche vorhanden. Deutlich zeigt sich, daß die Verkehrslage der beiden Städte weniger ausgeprägt, bzw. Zofingen lediglich Bezirkshauptort ist.

Waldenburg kann wegen seiner Lage lediglich im sekundären Erwerbssektor von relativer Bedeutung sein. Bezeichnenderweise ist hier der Anteil der industriell Berufstätigen gleich wie in Aarburg am höchsten, der Anteil der Dienstleistungsberufe jedoch noch deutlich kleiner als in Aarburg. Das enge Vordere Frenketal erlaubt zudem nur Industrien, die nicht allzu großer Areale bedürfen, und so ist hier in erster Linie, wie übrigens auch in den Gemeinden talabwärts, die Uhrenindustrie anzutreffen. Die Paßfuß-Funktion ist heute im Gegensatz zu früher kaum mehr ausschlaggebend.

Wiedlisbach besitzt selber keine großen oder für die Gemeinde charakteristischen Industrien. Die meisten in der Industrie Tätigen gehen als Wegpendler Richtung Solothurn, Wangen a. A. oder Oensingen -Klus. Daß in Wiedlisbach der eindeutig höchste Anteil der landwirtschaftlich Berufstätigen festzustellen ist, hängt mit der freien Lage und dem Landschaftstyp des nördlichen Bipperamtes zusammen.

Ähnliche Unterschiede ergeben sich zwangsläufig in Bezug auf die Seßhaftigkeit der Bevölkerung in den sechs Gemeinden. Entsprechend der starken Industrialisierung und dem damit verbundenen Bevölkerungsanstieg bzw. der Zuwanderung in den letzten Jahren hat Aarburg am wenigsten Einwohner, die auch hier geboren worden sind, nämlich nur $22 \%$; Heimatort und Wohnort sind sogar nur bei 5\% übereinstimmend. Fast genau gleich ist die Situation in Olten, da Bahndienst, Verkehrs- und Handelsberufe im allgemeinen einem stärkeren Wohnortswechsel ausgesetzt sind. Nur bei $27 \%$ stimmt der Wohnort mit dem Geburtsort und lediglich bei $6 \%$ der Wohnort mit dem Bürgerort überein. Dann folgt Liestal mit Anteilen von 32 bzw. 12\%. In Zofingen, das lange Zeit von allen Froburger Städten am einwohnerreichsten war, steigen die Zahlen auf $33 \%$ für Geburts-Wohnort und $16 \%$ für Heimat-Wohnort an. In Waldenburg betragen sie für die erste Gruppe $40 \%$, für die zweite aber nur $15 \%$, was wohl mit der stets nur geringen Einwohnerzahl zusammenhängt. Wiedlisbach hat hingegen nur $32 \%$ in der Gemeinde Geborene, jedoch die höchste Zahl, nämlich $26 \%$, von Leuten, bei denen
Wohnort und Heimatort identisch sind. Dieser Umstand muß sicherlich mit dem relativ noch starken bäuerlichen Anteil an der Gesamtbevölkerung in Verbindung gebracht werden.

\subsection{Die Wirtschaftsstruktur der Umgebungen}

Weitgehend übereinstimmend mit der Struktur der sechs Stadtgemeinden ist auch diejenige ihrer unmittelbaren Umgebungen. Die Durchschnitte der noch landwirtschaftlich Berufstätigen lauten wie folgt: Umgebung von Olten 9\%, Umgebung von Aarburg und Zofingen 13\%, Umgebung von Liestal $21 \%$, Umgebung von Wiedlisbach $21 \%$ und Umgebung von Waldenburg $25 \%$. Hier fällt auf den ersten Blick höchstens auf, daß die Umgebungen von Liestal und Wiedlisbach denselben landwirtschaftlichen Anteil besitzen, doch bleibt zu beachten, daß die Lage Liestals eine ganz andere ist als diejenige Wiedlisbachs. Liestal liegt im Tafeljura, wo der Strukturunterschied zwischen den Haupttal- und Tafel- oder Nebentalgemeinden beträchtlich ist; Tafelgemeinden grenzen unmittelbar an Liestal, liegen aber in einer völlig andern Wirtschaftslandschaft. So sind die Unterschiede zwischen den um Liestal liegenden Gemeinden recht groß. Die Haupttaldörfer Frenkendorf und Lausen besitzen nur $4 \%$, Füllinsdorf nur 6\% landwirtschaftlich Erwerbstätige, in den abgelegenen Dörfern Hersberg und Arisdorf zum Beispiel steigen die Zahlen auf 56 bzw. $42 \%$. Daß Oltens Umgebung am wenigsten landwirtschaftliche Bevölkerung aufweist, ist nicht verwunderlich. Von den 14 in diese Betrachtung einbezogenen Dörfern haben nur noch sechs über $10 \%$ landwirtschaftlich Berufstätige, Trimbach hat sogar nur 2\%, Wangen und Dullikon weisen noch $4 \%$ auf. Einzig in der Juragemeinde Hauenstein-Ifenthal ist ein Prozentsatz von 38 vorhanden. Diese Gemeinde hat zudem auch eine rückläufige Bevölkerungsbewegung zwischen 1950 und 1960. Einige der umliegenden Dörfer Oltens sind zu ausgesprochenen Wohn- und Vorortsgemeinden geworden, am deutlichsten Trimbach.

\subsection{Die wirtschaftlichen Einzugsgebiete}

Eine Betrachtung der Einzugsgebiete von Wiedlisbach und Waldenburg erübrigt sich, da kaum solche bestehen. Wir verzichten auch auf eine Darstellung des Einzugsgebietes von Aarburg und halten hier nur fest, daß einzig aus Rothrist $10 \%$ der dort wohnhaften Berufstätigen nach Aarburg zur täglichen Arbeit gehen und aus allen umliegenden Dörfern die Pendleranteile niedriger sind. Detailunter- 


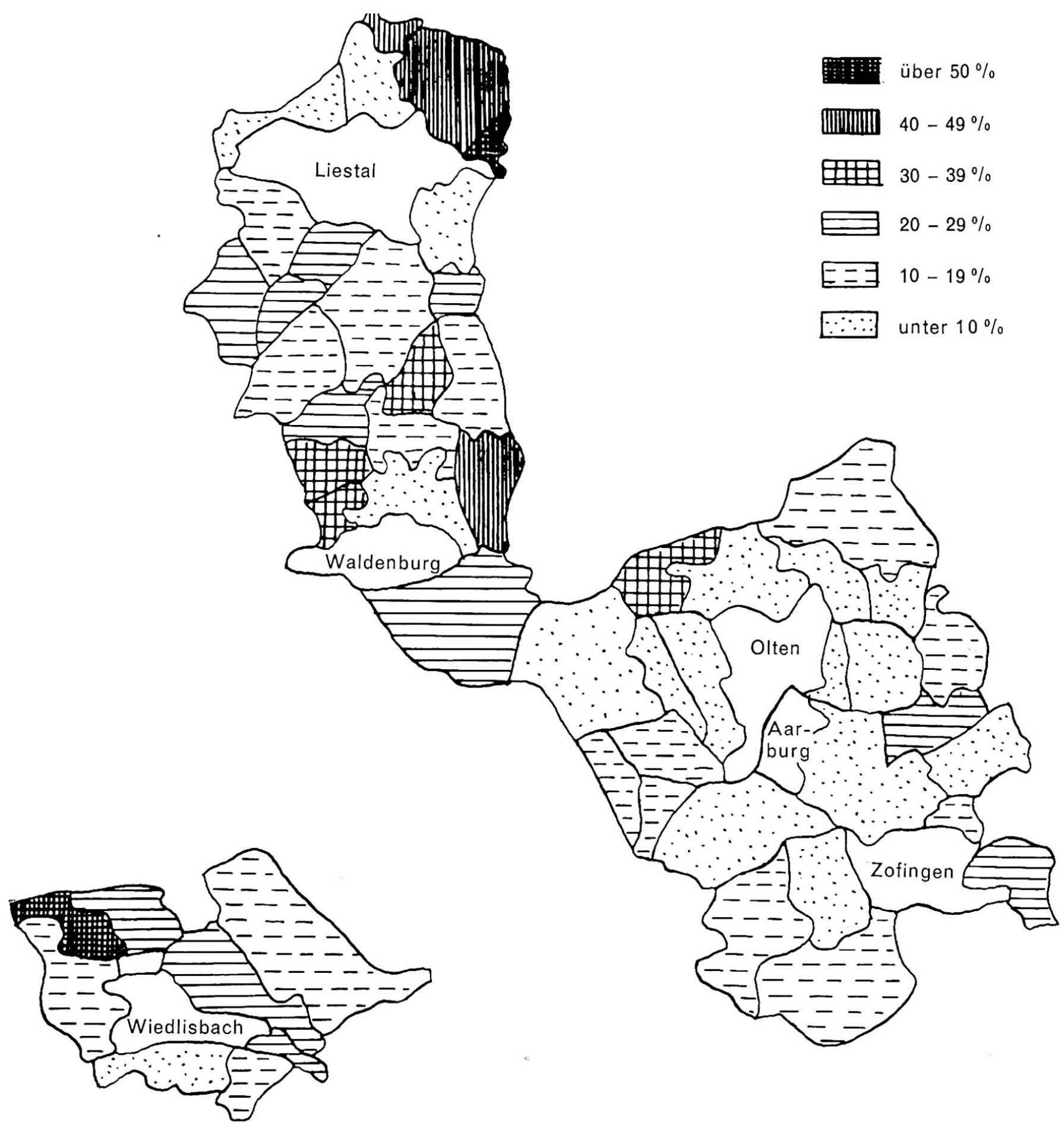

Fig. 11 Landwirtschaftlich Berufstätige in den umliegenden Gemeinden (1960).

suchungen für die Einzugsgebiete liegen für die Städte Olten und Zofingen vor, allerdings noch auf der Volkszählung von 1950 basierend $(23,24)$. Vergleiche mit den Zahlen von 1960 haben jedoch nur geringfügige und unwesentliche Veränderungen ergeben.

Eine genaue Bestimmung der Einzugs- oder Marktgebiete erfordert zahlreiche Einzeluntersuchungen aus diversen Bereichen des wirtschaftlichen Lebens. Die beiden erwähnten Untersuchungen über Olten und Zofingen zeigen jedoch, daß hier allein schon mit einer Analyse der Pendlerzahlen die Gebiete weitgehend und durchaus zuverlässig abge- steckt werden können, denn der Pendelverkehr ist wohl der deutlichste Ausdruck der ökologischen Relationen zwischen dem Zentrum und der Umgebung.

Ganz allgemein kann festgehalten werden, daß in Olten und Zofingen der Pendelverkehr beträchtliche Ausmaße angenommen hat und die Einzugsgebiete entsprechend groß sind. 1960 betrug zum Beispiel für Olten die Zahl der täglichen Zupendler 6755, welche aus über 190 verschiedenen Gemeinden stammten. In Zofingen arbeiten sogar mehr auswärtige als in der Gemeinde selbst wohnhafte Berufstätige. 

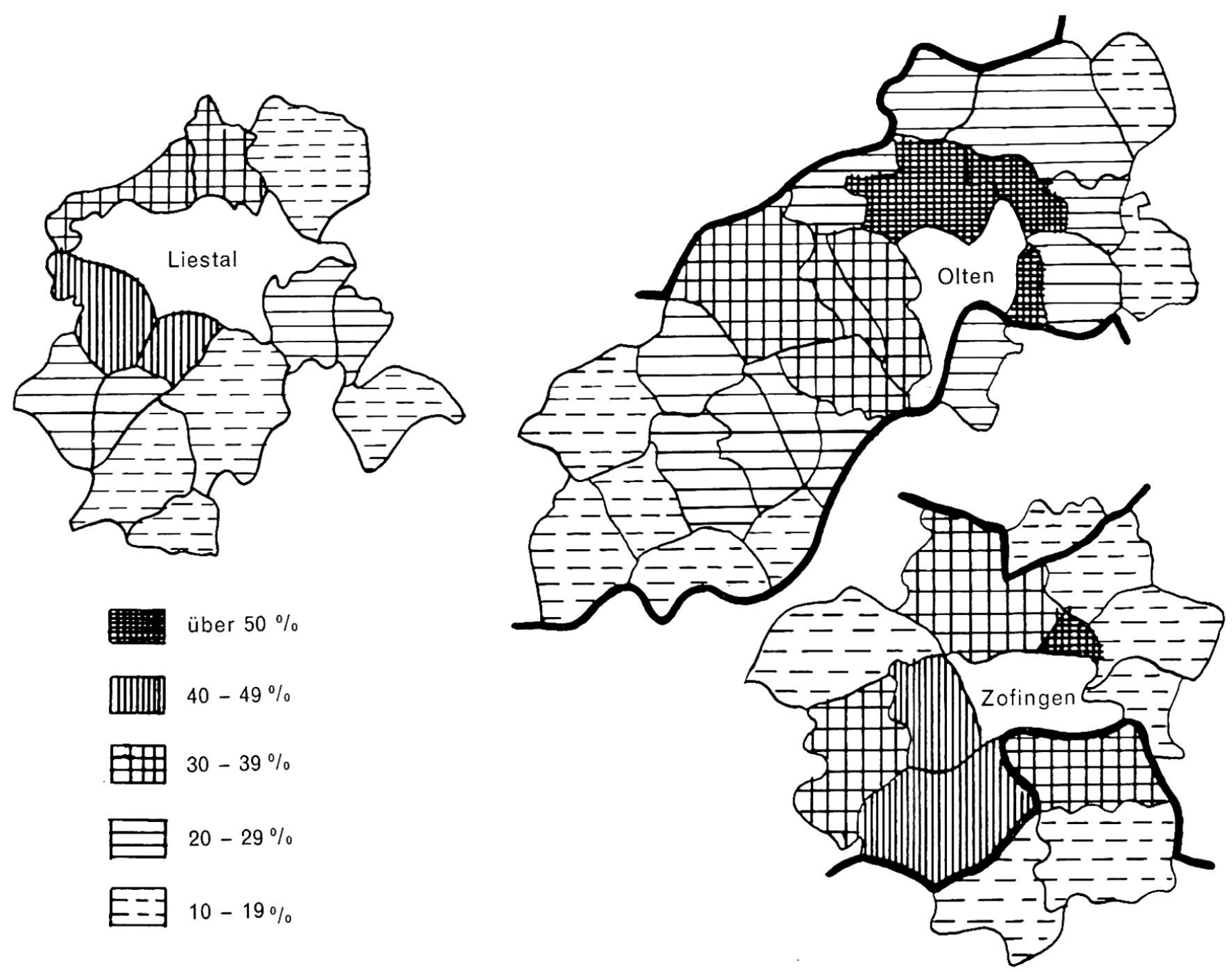

Kantonsgrenzen

Fig. 12 Pendler-Einzugsgebiete. Wegpendler in \% der wohnhaften Berufstätigen (1960).

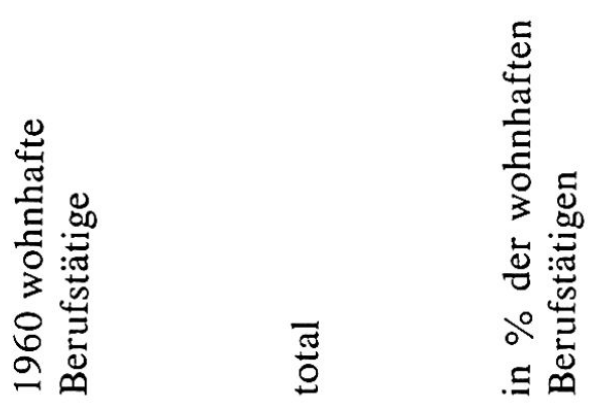

Zupendler 1960

$\begin{array}{lrrr}\text { Olten } & 9570 & 6755 & 70 \\ \text { Zofingen } & 4293 & 5162 & 120 \\ \text { Liestal } & 4763 & 3164 & 66 \\ \text { Aarburg } & 2641 & 1231 & 46 \\ \text { Waldenburg } & 583 & 405 & 69 \\ \text { Wiedlisbach } & 771 & 56 & 7\end{array}$

Das ausgedehnteste Zupendlergebiet besitzt Olten. 7 Gemeinden mit zusammen 15272 Einwohnern (1960) schicken über $30 \%$ der eigenen Berufstätigen nach Olten und aus 4 Gemeinden mit zusammen 10857 Einwohnern fahren über $75 \%$ der im
Dorf wohnenden Wegpendler nach Olten. Das voll verbundene Einzugsgebiet oder das nahe Umland umfaßt mit Sicherheit die Dörfer Trimbach, Winznau, Starrkirch-Wil, Wangen, Rickenbach, Hägendorf, Kappel, Härkingen und Lostorf (24). Dazu kommen als weiteres Umland oder Hinterland (Intensivzone) noch 17 Ortschaften, die größtenteils im Westen der Stadt, im Gäu liegen. Die Gesamteinwohnerzahl der beiden Gebiete, die Stadt Olten mitgezählt, beläuft sich auf 50000 bis 60000 Menschen. Hierbei ist zu bemerken, daß Oltens Einzugsgebiet in mancherlei Hinsicht eingeengt ist. Gegen Norden erweist sich der Jura als Schranke und nur die beiden Hauensteintunnels verbinden noch ein paar Baselbieter Gemeinden äußerst lose mit Olten. Gegen Osten bilden der Arbeits- und Industrieort Schönenwerd und gleich daran anschließend die Stadt Aarau einen Abschluß und gegen Süden macht das Einzugsgebiet in gewisser Hinsicht bereits an der Stadtgrenze halt, die hier auch Kantonsgrenze ist und in die Industrieregion von Aarburg-Oftringen-Zofingen-Rothrist hin- 
überführt. Einzig Richtung Westen stellen sich keine größern Hindernisse in den Weg. Zwar erlebt das Gäu seit einiger Zeit und zu einem guten Teil als Folge des Nationalstraßenbaus und der dort erfolgenden Verschlingung von $\mathrm{N} 1$ und $\mathrm{N} 2$ eine eigene, überaus intensive Industrialisierung, womit es sich in einigen Belangen von Olten zu lösen beginnt.

Im Verhältnis zur Einwohnerzahl recht ausgedehnt ist auch das Einzugsgebiet von Zofingen. Aus 5 Gemeinden mit zusammen 15165 Einwohnern (1960) kommen über $30 \%$ der dort wohnhaften Berufstätigen tagtäglich nach Zofingen und aus 2 Dörfern mit zusammen 3243 Bewohnern fahren über $75 \%$ der Wegpendler nach Zofingen. Zum nahen Umland können auf alle Fälle Mühletal und Strengelbach gezählt werden, dann aber auch Brittnau, Wikon und Oftringen. Als weiteres Umland kämen noch Reiden und Vordemwald hinzu, alles Dörfer innerhalb der 15-Minuten-Isochronen. Eventuell ergänzen ließe sich dieses Gebiet noch Richtung Safenwil und Uerketal und gegen Langnau bei Reiden und Rothrist. Zofingens Einzugsgebiet ist weitgehend durch Form und Verlauf des untern Wiggertales bestimmt. Die Dörfer westlich und östlich davon liegen auf oder sogar hinter den das Tal begleitenden Molassehügelzügen und sind teilweise nur mit kleinen Straßen mit ansehnlichen Steigungen mit dem Haupttal verbunden. Dann ist aber auch hier, ähnlich wie bei Olten, zu berücksichtigen, daß die südliche Gemeindegrenze von Zofingen zugleich Kantonsgrenze ist und die nördlich anschließenden aargauischen Gemeinden im Grenzraum der beiden allzu nahen Zentren Zofingen und Olten liegen.

Nur nach Pendlerzahlen bestimmt, ergibt sich für Liestal eindeutig das kleinste Gebiet, und dies obwohl Liestal mehr Einwohner als Zofingen hat und zudem noch Kantonshauptort ist. Nur 4 Gemeinden mit zusammen 3454 Einwohnern (1960), 2 davon im Kanton Solothurn gelegen, haben mehr als 30\% in Liestal Erwerbstätige, nämlich Lupsingen, Nuglar-St. Pantaleon, Büren und Füllinsdorf. Dabei fällt erst noch auf, daß keine einzige davon $50 \%$ erreicht, während es bei Olten deren 3 und bei Zofingen 1 sind. Um Liestal gibt es kein einziges Dorf, aus dem über $75 \%$ der Wegpendler in Liestal arbeiten. Am ehesten noch zum weitern Umland zu zählen wären Seltisberg, Bubendorf, Frenkendorf und Lausen, von denen das letzte aber selber wiederum über viele eigene Arbeitsplätze verfügt. Das Liestaler Einzugsgebiet ist weitgehend durch die Gestalt des Tafeljuras und dessen Talsystem bedingt. Liestal liegt 329 Meter über Meer, die angrenzenden Dörfer Hersberg und Nuglar zum Beispiel jedoch 510 bzw. 487 Meter. Zudem reicht der Sog der nahen Großstadt Basel bereits bis nach Liestal hinauf, wogegen für Olten und Zofingen die zentralen Orte der höchsten Stufe doch wesentlich weiter entfernt sind und vor allem Olten mit seiner einzigartigen Verkehrslage sowohl im Bereich von Basel, Zürich und Bern liegt.

Unterschiedlich sind auch die Zahlen der Wegpendler:

Wegpendler in \% der wohnhaften Berufstätigen (1960)

Wiedlisbach 46

Aarburg

41

Waldenburg

19

Olten

18

Liestal

14

Zofingen

Wiedlisbach besitzt keine eigenen großen Industrien, Aarburg liegt zwischen den beiden Zentren Zofingen und Olten und Waldenburgs Wegpendler dürften in erster Linie in die Uhrenindustrie der Nachbardörfer im gleichen Tal fahren. Die überraschend hohe Zahl für Olten ist mit den günstigen Schnellzugsverbindungen nach Basel, Zürich, Luzern, Bern und Biel (überall Fahrzeiten unter 60 Minuten) zu erklären. Die geringen Zahlen von Liestal und Zofingen schließlich zeigen deutlich die relativ hohe Zentralität auch dieser beiden Städte.

\subsection{Die Verkehrslage}

Seit der Entstehungszeit der Froburger Städte im Mittelalter erfuhren die großen schweizerischen Verkehrslinien einige wesentliche Verlegungen, welche für einen Teil der Städte beträchtliche Folgen hatten. Die erste Neuorientierung ergab sich mit dem Eisenbahnbau, der vor allem Waldenburg und Wiedlisbach in Mitleidenschaft gezogen hat. Mit dem Bau der beiden Hauensteintunnels Richtung Olten ging der Verkehr über den Obern Hauenstein, der einst einer der wichtigsten Juraübergänge überhaupt war, vorübergehend stark zurück. Waldenburg hatte in der Folge zwischen 1870 und 1880 eine geringfügige Bevölkerungsabnahme zu verzeichnen, die in Langenbruck auf der Paßhöhe Z'vischen 1850 und 1910 sogar rund 150 Personen betrug. Auf der solothurnischen Südseite des Überganges nahm Holderbank von 1850 bis 1888 ebenfalls um über 100 Einwohner ab. Beim Bau der Jurafußlinie Olten-Biel in den Siebzigerjahren wurde bei der Linienführung Wiedlisbach nicht berücksichtigt: die Bahn wich von Niederbipp an Richtung Süden nach Wangen a. A. aus, wobei die durch die große Endmoräne bedingte Geländeform ebenfalls maßgebend gewesen sein dürfte. Die an sich schon recht kleinen Städte Wiedlisbach und 
Waldenburg wurden somit vorübergehend vom groBen Transitverkehr umfahren und bekamen in der Folgezeit lediglich Lokalbahnanschlüsse: Wiedlisbach liegt an der in ihrem Fortbestand stets wieder umstrittenen Schmalspurlinie Solothurn-Niederbipp und Waldenburg ist Endstation der Schmalspurbahn, welche von Liestal aus das Vordere Frenketal bedient. Im Zeitalter des Automobilverkehrs hat sich die Lage sodann für beide Orte wieder gebessert. Beide liegen an der Straße Basel-Bern, und in Wiedlisbach hat sich der Automobilist zu entscheiden, ob er nach Bern die Route über Wangen oder über Solothurn wählen will. Die engen Gassen von Wiedlisbach und Waldenburg bewirken gelegentlich an dieser Strecke starke Verkehrsstockungen. Seit dem Juni 1966 hat sich jedoch für Wiedlisbach die Situation insofern geändert, als daß nun die Nationalstraße Nr. 1 südlich des Städtchens vorbeiführt und dadurch den vorher intensiven Durchgangsverkehr merklich reduziert. 18 Auch Waldenburg steht vor einer neuerlichen Änderung seiner Verkehrssituation. Vorläufig erreicht der von Basel kommende Autofahrer die N1 erst in Oensingen und muß also noch immer den Engpaß Waldenburg passieren. Mit der Eröffnung des Belchentunnels jedoch wird auch der Verkehr über den Obern Hauenstein zurückgehen, wenn auch kaum anzunehmen ist, daß dadurch eine Wiederholung des wirtschaftlichen Rückgangs wie zur Zeit des Eisenbahnbaues erfolgen wird.

Liestal kam beim Bahnbau an die Linie Basel-Olten (alter und neuer Tunnel) zu liegen. Es besitzt seiner Zentralität entsprechend auch einige Schnellzugshalte. Zudem ist es Ausgangspunkt der Waldenburgerbahn und einiger Postautolinien. Im Straßenverkehr blieb es bis heute Verteiler für die Route Basel-Oberer Hauenstein-Bern-Westschweiz und Basel-Unterer Hauenstein-Luzern-Gotthard, so daß der in beiden Richtungen durch Liestal rollende Verkehr zuweilen erschreckende Ausmaße annimmt und die Stadt wohl den gefürchtetsten Engpaß überhaupt zwischen Basel und Bern bzw. Luzern darstellt. Auch die eher improvisierten Umfahrungen der Altstadt führen auf schmalen Straßen durch überbautes Gebiet, so daß erst mit der Autobahn, die Liestal über Arisdorf Richtung Eptingen-Belchentunnel umgehen wird, sich die Straßenverhältnisse in Liestal merklich bessern werden. Allerdings wird bis zur Fertigstellung der Nationalstraßen in diesem Raum noch einige Zeit verstreichen.

Olten hat von allen Froburger Städten noch immer und in jeder Beziehung eindeutig die beste Verkehrslage. In den Fünfzigerjahren des 19. Jahrhunderts aus dem zähen Ringen um den Eisenbahnknotenpunkt der Centralbahngesellschaft als Sieger hervorgegangen, besitzt die Stadt heute einen der bedeutendsten Bahnhöfe der Schweiz. Úber 900 Züge aller Art fahren im Tag ein und aus. Sechs Eisenbahnlinien strahlen von Olten aus, von denen nur diejenige durch den alten Hauensteintunnel nach Läufelfingen-Sissach lokal-regionale Bedeutung hat. Die andern sind alle doppelspurige Schnellzugsund Güterlinien, und die minimalen Fahrzeiten sind äußerst gering: nach Zürich 46 Minuten, nach Luzern 41 Minuten, nach Basel 28 Minuten, nach Bern 49 Minuten und nach Biel 44 Minuten. Verschiedene Berechnungsmethoden haben ergeben, daß Olten im schweizerischen Eisenbahnverkehr von allen Städten die beste Erreichbarkeit aufweist (24). Dasselbe gilt für den Straßenverkehr, für welchen sich in der Stadt die Straße Basel-Luzern-Gotthard mit derjenigen von Zürich-Aarau-SolothurnBiel dem Jurasüdfuß entlang kreuzt. Zudem führt die Hauptstraße Bern-Zürich auch nur wenige Kilometer südlich der Stadtgrenze vorbei. Olten hat somit zusätzlich zum Binnen- und Vorortsverkehr vor allem einen überaus starken Durchgangsverkehr in verschiedenen Richtungen. Alle Verkehrstypen und -richtungen sammeln sich auf der Bahnhofbrücke, wo täglich gegen 30000 PWE passieren, eine Zahl, die in der Reise- und Feriensaison noch beträchtlich erhöht wird. Ein kürzlich ausgearbeiteter Verkehrsplan sah deshalb eine zweite Aarebrücke vor (20). Bestimmt wird mit der nächstens erfolgenden durchgehenden Eröffnung der Autobahnen der Verkehr auf Stadtgebiet ebenfalls nachlassen, die Verkehrslage Oltens im Straßenverkehr wird aber erneut aufgewertet werden, denn $\mathrm{N} 1$ und N2 werden sich nur $8 \mathrm{~km}$ südwestlich der Stadt zwischen Egerkingen und Härkingen verschlingen. Damit wird Oltens Lage im Eisenbahnverkehr eine Parallele im Straßenverkehr bekommen. Daß Olten schon längst gelegentlich als Konferenzstadt bezeichnet wird, ist angesichts seiner heutigen Verkehrslage und Erreichbarkeit auf Schiene und Straße nicht verwunderlich.

Aarburg ist mit dem Nachbarort Olten durch eine der meistbefahrenen Eisenbahnlinien der Schweiz verbunden, denn erst in Aarburg gabeln sich die Linien nach Luzern und Bern. ${ }^{19}$ Schnellzugshalte gibt es hier zwar nicht; diejenigen von Olten und Zofingen liegen aber sehr nahe, und zudem bestehen Autobuskurse nach diesen beiden Richtungen. Im Straßenverkehr bildet Aarburg seiner Klusenlage zufolge ebenfalls einen unliebsamen Engpaß an der Route Basel-Luzern, von der hier noch eine Verbindung nach Rothrist zur N1 abzweigt. Auch dadurch, daß Aarburg schon jetzt sehr nahe am Kreuz der Hauptstraßen 1 und 2 liegt und auch die Autobahnverschlingungen nicht weit entfernt sind, erklärt sich die starke Industrialisierung der Region in letzter Zeit weitgehend.

Zofingen besitzt als Zentrum des Wiggertales und Standort einiger bedeutender Industrien ebenfalls einige Schnellzugshalte im Eisenbahnverkehr an der 
Linie Basel-Olten-Luzern. In Zofingen zweigt davon die Strecke über den Striegel nach Aarau ab, das letzte vollendete Teilstück der ehemaligen $\mathrm{Na}$ tionalbahn. Hätte das Projekt Erfolg gehabt, wäre Zofingen ebenfalls zu einem Knotenpunkt und damit Konkurrenten Oltens geworden; der Konkurs der Gesellschaft im Jahre 1880 hat jedoch für Zofingen ansehnliche Schulden zur Folge gehabt. Der Straßenverkehr ist in erster Linie bestimmt durch den Süd-Nord-Verlauf des Wiggertales. Die beiden Verkehrsströme nach Luzern und Olten-Basel bewegen sich östlich und westlich um die Altstadt herum, vor allem unter Benutzung der ehemaligen Stadtgrabenareale. Im übrigen profitieren Zofingen und seine Industrien ebenfalls von der Nähe des Bahnhofes Olten und des Straßenkreuzes von Oftringen und der Autobahnen.

\subsection{Baulich-räumliche Entwicklung und Agglome- rationen}

$\begin{array}{ll}\text { Bewohnte } & \text { Zunahme in \% } \\ \text { Gebäude 1960 } & 1947-1960\end{array}$

$\begin{array}{lrl}\text { Olten } & 2692 & 45 \\ \text { Aarburg } & 817 & 73 \\ \text { Zofingen } & 1366 & 44 \\ \text { Wiedlisbach } & 285 & 30 \\ \text { Waldenburg } & 245 & 19 \\ \text { Liestal } & 1546 & 47\end{array}$

Die Zahlen gehen ungefähr parallel mit denjenigen der Bevölkerungsbewegung; Typ und Struktur der sechs Städte spiegeln sich eher in den folgenden Angaben über die Verbreitung der Einfamilienhäuser:

$\begin{array}{ll}\text { Bewohnte } & \text { davon \% in } \\ \text { Wohnungen } 1960 & \text { Einfamilienhäusern }\end{array}$

$\begin{array}{lrl}\text { Olten } & 6168 & 19 \\ \text { Aarburg } & 1476 & 31 \\ \text { Zofingen } & 2545 & 24 \\ \text { Wiedlisbach } & 450 & 26 \\ \text { Waldenburg } & 386 & 33 \\ \text { Liestal } & 2673 & 30\end{array}$

Olten hat seit 1850 entschieden die stärkste baulichräumliche Entwicklung durchgemacht, so daß bald einmal das überbaubare Areal aufgebraucht sein wird. Schon heute nähern sich die Außenquartiere fast überall den naturbedingten Grenzen an den Wald- und Jurarändern. Olten galt lange Zeit als ausgesprochene Garten- und Einfamilienhausstadt, was wesentlich mit der Berufsstruktur der Bevölkerung im Zusammenhang gestanden haben dürfte. Heute ist jedoch der Platzmangel derart, da $\beta$ immer mehr ältere Einfamilienhäuser im Stadtzentrum

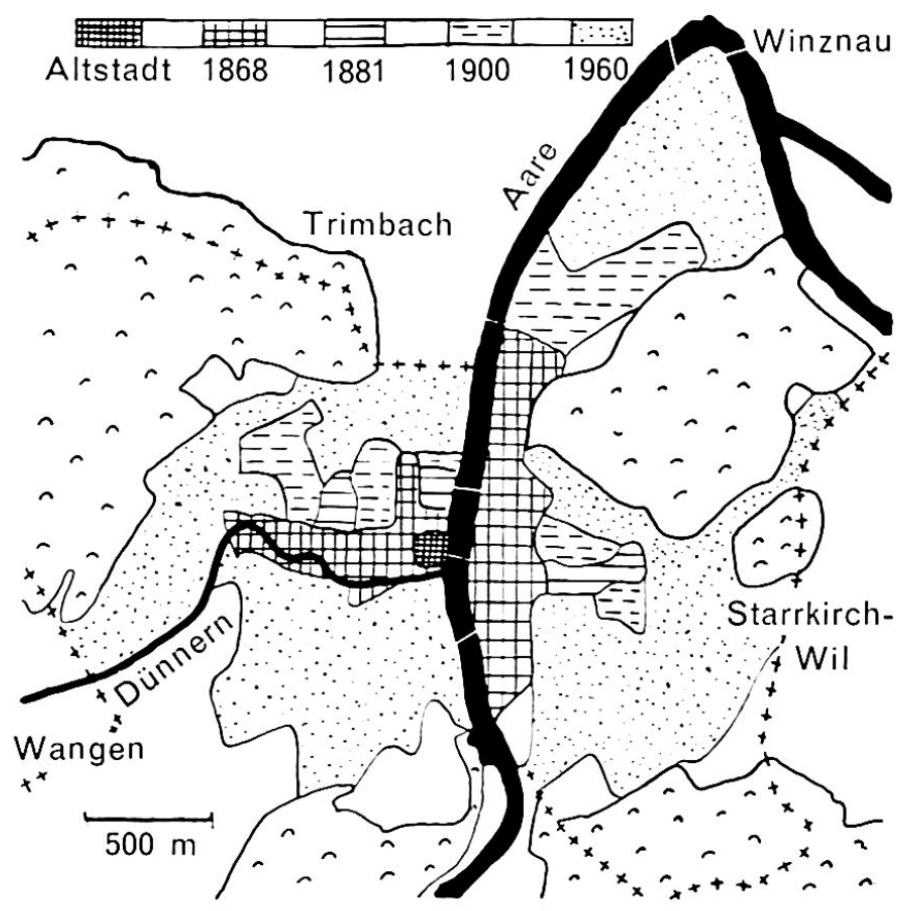

Fig. 13 Siedlungsausdehnung Oltens 1868-1960 (inkl. Industrie- und Bahnareale).

und auch schon in den Außenquartieren Wohnblocks und Hochhäusern weichen müssen, was sich in den obigen Zahlen in dem mit Abstand geringsten Prozentsatz der Wohnungen in Einfamilienhäusern äußert. Zudem ist zu bedenken, daß heute Oltens Bevölkerung infolge der Verkehrslage noch vermehrt einem ständigen Wechsel unterworfen ist. Auch diese Tatsache wirkt sich eher auf den Neubau von Blocks als auf denjenigen von Einfamilienhäusern aus. Neue Einfamilienhäuser sind in den letzten Jahren nur noch wenige erstellt worden; solche finden sich dagegen entsprechend häufig in den Dörfern des Einzugsgebietes. Das baulich-architektonische Aussehen Oltens ist heute als recht modern zu bezeichnen. Eine teilweise fast großstädtisch anmutende Geschäftszone beginnt die kleine, fast kreisrunde froburgische Altstadt mit der Holzbrükke immer mehr einzukreisen und zu erdrücken.

In den zentralen Quartieren ist die bauliche Entwicklung Oltens weitgehend durch den Bahnbau bestimmt worden. Der vom ehemaligen Stadtkern relativ weit abgelegene Bahnhof und die Aarebrükke davor verursachten eine Schwerpunktsverlagerung und Umorientierung des Verkehrsflusses: die früher vom Verkehr durchzogene Altstadtachse verläuft west-ost, die Bahnhof und Geleiseachse jedoch kam nord-süd zu liegen und befand sich jenseits der Aare und in bis anhin noch kaum überbautem Gebiet. So führt der Durchgangsverkehr seit 1882 über die Bahnhofbrücke und umfährt die Altstadt, aus der in der Folgezeit immer mehr zentrale Dienste und öffentliche Funktionen abgewandert sind. Der Verlauf der Bahnlinien und die Lage 


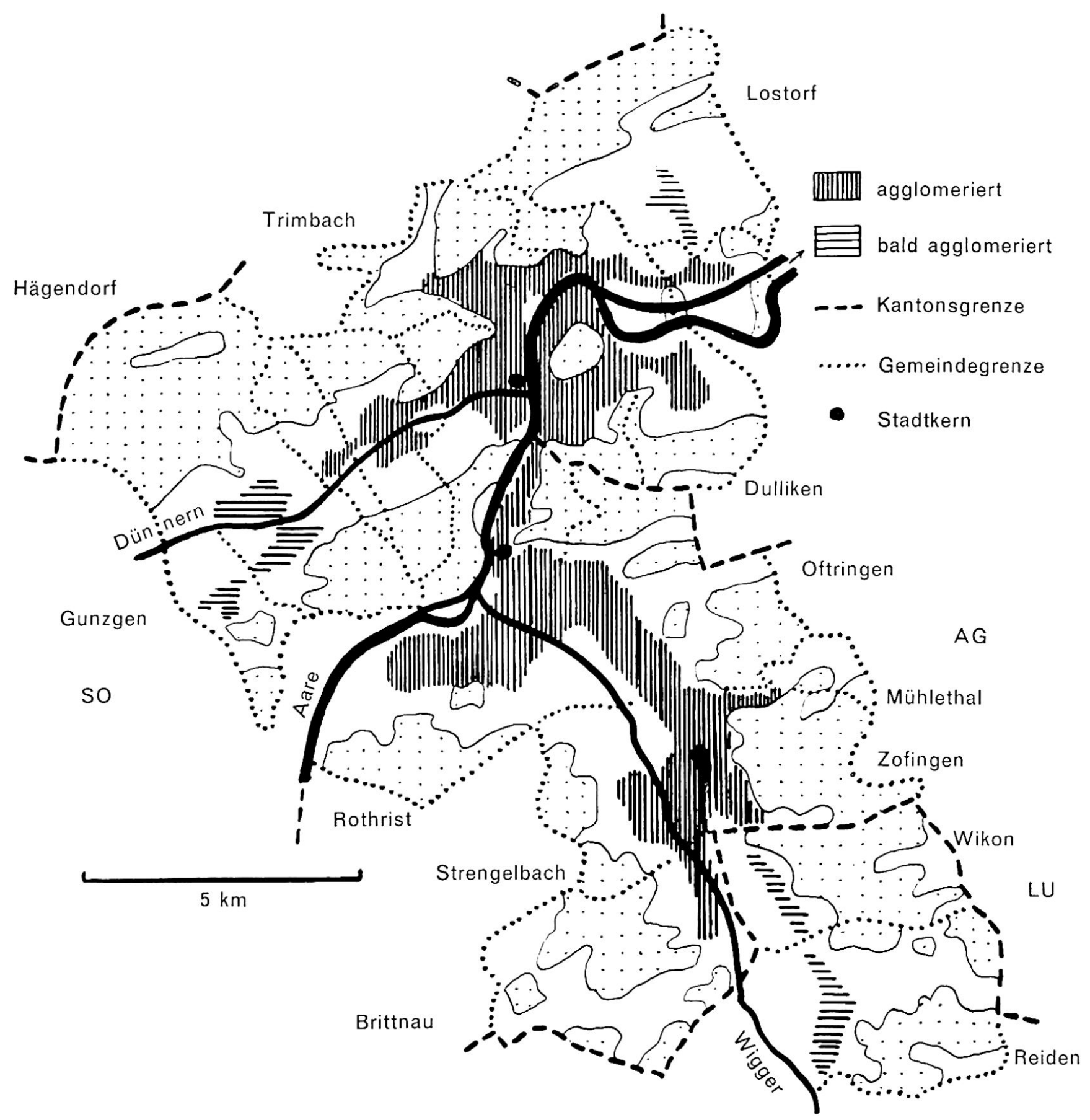

Fig. 14 Agglomeration Olten-Aarburg-Zofingen. Bewaldete Gebiete im Norden am Jurasüdhang, in der Mitte an der Born-Engelberg-Kette, im Sü den auf den Molassehöhen des Wiggertales.

der beiden Bahnhöfe bewirkten, daß sich die Industrien in Schienennähe niederließen und von einigen Ausnahmen abgesehen relativ geschlossene Industriezonen zwischen Hardwald und Aare im Nordosten und in der Dünnernausräumung im Westen entstanden sind. Für die Wohnquartiere verblieben die recht gut exponierten Hänge gegen die Waldränder an den Juraketten im Norden und Süden der Stadt.

Aarburg hat schon früh eine bauliche Entwicklung am Südfuß des Burgfelsens erfahren, wo sich an einem von der Wigger abgeleiteten Kanal eine Reihe von Gewerbebetrieben und in der Folge auch Industrien festgesetzt hatten. Auch in Aarburg führte der Eisenbahnbau zu einer Umorientierung in der bau- lichen Entwicklung. Aus Gelände- und eisenbahntechnischen Gründen (Gabelung der Linien nach Luzern und Bern, Gemeinschaftsbahnhof Aarburg/ Oftringen) mußte der Bahnhof ebenfalls relativ weit weg vom alten Stadtkern südöstlich des Burgfelsens gebaut werden. Eine erste Industriezone entstand demnach in jener Gegend, und sowohl von Aarburg als auch von Oftringen wuchsen dann auch die Wohnquartiere immer mehr Richtung Bahnhof und Wiggerebene. Das zur Gemeinde Aarburg gehörende Areal ist dort allerdings sehr klein, so daß neuestens immer mehr auch die Aareklus bis zur Grenze Oltens mit Industrie- und Wohnbauten überdeckt wird. Aarburg teilt sich demnach deutlich in zwei durch den Sporn des Burgfelsens recht sichtbar ge- 
trennte Hälften, von denen die eine in mancher Beziehung eher nach Olten, die andere jedoch nach Zofingen orientiert ist.

Zofingen, das im Mittelalter die größte überbaute Fläche besessen hatte, hat sich infolge des Verlaufes der Gemeindegrenzen in seinem räumlichen Wachstum an die Wiggerebene östlich des Flusses halten müssen. Bahnhofareal und Bahnlinien wirkten dabei weitgehend zonenbildend, indem sie die Industriebauten von der Gegend der Altstadt fernhielten. Städtebaulich von ganz besonderem Reiz und Vorteil ist die Tatsache, daß es in Zofingen gelang, übrigens ganz ähnlich wie in Solothurn, die Zone östlich der Altstadt als Grünfläche zu erhalten und das parkartige Gelände lediglich mit ein paar öffentlichrepräsentativen Gebäuden zu versehen. Die Wohnquartiere erstrecken sich nördlich und südlich des Stadtkerns in der Wiggerebene und in neuerer Zeit auch immer mehr hinauf bis zu den Waldrändern am ostwärts anschließenden Molassehang. Auch Zofingens Ausdehnungsmöglichkeiten sind heute vor allem wegen der allzu engen Gemeindegrenzen im Norden, Westen und Süden bereits beschränkt.

Seit einiger Zeit sind Olten, Aarburg und Zofingen zusammen mit den umliegenden Gemeinden zu einer Agglomeration verschmolzen, was umso erstaunlicher ist, als sich mittendrin die allerdings klusendurchbrochene Born-Engelberg-Antiklinale erstreckt. Als Maß zur Beurteilung, ob zwei Nachbargemeinden als agglomeriert $\mathrm{zu}$ betrachten seien, wird eine Häuserdichte von 15 pro 100 Meter StraBenlänge angenommen (5). Demnach müssen zur Agglomeration Olten-Aarburg-Zofingen gezählt werden: Olten, Trimbach, Winznau, Obergösgen, Starrkirch-Wil, Dulliken, Wangen, Rickenbach, Aarburg, Rothrist, Oftringen, Mühletal, Strengelbach, Brittnau und Zofingen. Bald einmal agglomeriert werden sein im Raum Olten: Lostorf, Hägendorf, Kappel und Gunzgen, im Raum Zofingen: Wikon und Reiden. Ohne die zuletzt genannten Orte bereits mitzuzählen, ergibt sich (1960) für die gesamte Agglomeration der drei ehemaligen Froburger Städte eine Einwohnerzahl von 80000 bis 90000 Menschen. Diese verteilen sich auf zwei und bald einmal auf drei verschiedene Kantone, was die dringend notwendige Regionalplanung erschwert, aber gerade dadurch umso nötiger macht.

Liestals räumliche Entwicklung erfolgte rund um die Altstadt herum, ergolzab- und aufwärts und hinein ins Tal des Orisbaches. Die Landschaftsform des Tafeljuras legt hier eine gewisse Beschränkung auf und hat auch zur Folge, daß die Agglomeration Liestal kleiner ist und sich langgezogen nur an den Talboden der Ergolz halten kann. Als mit Liestal agglomeriert $\mathrm{zu}$ betrachten sind heute erst Lausen, Frenkendorf und Füllinsdorf, was zusammen rund 20000 Einwohner ausmacht. Hingegen dürfte der
Zeitpunkt nicht mehr fern sein, wo diese Agglomeration sich mit derjenigen von Basel verschmelzen wird.

Auch in Waldenburg ist die räumliche Ausdehnung durch die Lage eingeengt. Neue Quartiere entstanden talaufwärts und talabwärts um die Endstation der Waldenburgerbahn, wo aber gleich die Gemeinde Oberdorf beginnt, die als mit Waldenburg längs Straße und Frenke agglomeriert zu betrachten ist. In absehbarer Zeit wird die noch kleine, langgezogene Agglomeration mit Niederdorf, eventuell auch mit Hölstein vergrößert werden, womit sich vier ausgesprochene Straßensiedlungen längs einer Strecke von rund $7 \mathrm{~km}$ vereinigt hätten.

Wiedlisbach endlich hat eine geringfügige, lockere Überbauung längs der Zufahrtsstraßen und am nördlich anschließenden Jurahang erhalten. Eine Berührung mit einer Nachbargemeinde ist noch nirgends erfolgt, wobei erst noch zu bemerken wäre, daß gegen Süden Richtung Wangen a. A. einer derartigen Tendenz Aare und Aarekanal und neuerdings auch die Autobahn entgegenwirken.

\section{Literatur und Quellen}

Auf eine Aufzählung der umfangreichen lokalen, vor allem lokalhistorischen Literatur wird verzichtet. Aufgeführt sind nur Werke vorwiegend geographisch-kartographischer Art, die für den vorliegenden Überblick von Bedeutung waren.

\section{Literatur}

1 Ammann, H.: Die froburgischen Städtegründungen. Festschrift Nabholz. Zürich 1934.

2 Beck, H.: Glazialmorphologische Untersuchungen in der Gegend von Solothurn. Diss. Fribourg 1957.

3 Bütler, K.: Die wirtschaftliche Entwicklung der Stadt Liestal. Bern 1951.

4 Burckhardt, G.: Basler Heimatkunde. Basel 1933.

5 Carol, H.: Begleittext zur wirtschaftsgeographischen Karte der Schweiz, Geogr. Helv. I, 1946.

6 Disteli, M.: Aarburg, ein Beitrag zur Geographie einer Schweizer Kleinstadt. Diss.ETH, Zürich 1954.

7 Eidg. Volkszählungen, Bände Solothurn, Aargau, Baselland, Bern, Luzern und Pendelwanderung. Bern 1960 ff.

8 Felchlin, E.: Olten als Industriestandort. Diss. Basel 1956.

9 Flugbild der Schweizer Stadt. Bern 1963. Enthält. u. a.:

9a Boesch, H.: Stadt und Verstädterung. Zur Geographie der Schweizer Stadt.

9b Hofer, P.: Die Stadtgründungen des Mittelalters zwischen Genfersee und Rhein.

10 Geographisches Lexikon der Schweiz. Neuenburg 1902/1910. 
11 Gutersohn, H.: Geographie der Schweiz. Bd. 1 Jura. Bern 1958.

12 Häfliger, E.: Das römische Olten. Festschrift Tatarinoff. Solothurn 1938.

13 Heitz, F.: Aarburg. Schweizer Heimatbücher Bd. 124. Bern 1965.

14 Historischer Atlas der Schweiz. Aarau 1951.

15 Historisch-biographisches Lexikon der Schweiz. Neuenburg 1924.

16 Rahn, J. R.: Die mittelalterlichen Kunstdenkmäler des Kantons Solothurn. Zürich 1893.

17 Siegrist, A.: Zofingen. Schweizer Heimatbücher Bd. 43. Bern 1952.

18 Sigrist, H.: Aus der Geschichte des ehemaligen Städtchens Klus. Jurablätter 29, 1967.

19 Stähelin, F.: Die Schweiz in römischer Zeit. Basel 1948.

20 Spring, A.: Verkehrsplanung Olten. Bern 1965. 21 Walliser, P.: Das Stadtrecht von Olten, anhand der froburgischen Stadtrechtsfamilie. Olten 1951.

22 Wiesli, U.: Balsthal und seine Täler, eine Siedlungs- und Wirtschaftsgeographie. Diss. Bern 1951. 23 - Beiträge zur Bestimmung der wirtschaftlichen Einzugsgebiete der Städte Grenchen und Zofingen. Versuch einer Klassenarbeit. Geogr. Helv. 1959.

24 - Olten, ein Beitrag zur Geographie der Schweizer Stadt. Mitt. d. Natf. Ges. Solothurn, 20. Heft, 1961.

\section{Karten}

25 Landeskarten der Schweiz $1: 25000$. Blätter 1068 Sissach, 1088 Hauenstein, 1107 Balsthal, 1108 Murgenthal, 1109 Schöftland.

26 Huene von, F.: Geologische Karte von Liestal und Umgebung. In Verh. d. Natf. Ges. Basel, 12. Bd., 1900.

27 Mühlberg, F.: Geologische Karte des Hauensteingebietes $1: 25$ 000, mit Profiltafel und Erläuterungen. 1915.

28 - und Niggli, P.: Geologische Karte des Gebietes Roggen-Born-Boowald, 1:25 000. Mit Erläuterungen. 1911.

29 Niggli, P.: Geologische Karte von Zofingen, $1: 25$ 000. Mit Erläuterungen. 1912.

\section{Anmerkungen}

1 Heute sind nur noch ein paar Grundmauern sichtbar, die den Grundriß der einst ansehnlichen Burg erkennen lassen. Konservierungsarbeiten sind kurz vor dem Zweiten Weltkrieg begonnen worden und harren längst einer Fortsetzung.

2 Unsere historische Zusammenfassung stützt sich in erster Linie auf die grundlegende Arbeit Ammanns (1).

3 Heute meist Klus genannt. Das Städtchen scheint allerdings nicht von den Froburgern direkt gegründet worden zu sein, da sie hier nie irgend welche Rechte geltend machten, sondern nach ihrem Vorbild von den Grafen von Falkenstein (18).

4 Vor allem auf der Nordseite. Pläne des römischen Castrums finden sich in Lit. 12 und 19.
5 Plan und Beschreibung in Lit. 16. Das Areal, das die kleine Stadt trug, ist auf der Landeskarte $1: 25$ 000, Blatt 1108 Murgenthal, noch deutlich sichtbar.

6 In Lit. 13 wird allerdings die Vermutung ausgesprochen, die Stadt könnte nördlicher, also innerhalb der Klus, gelegen haben. Damit wäre sie auch größer gewesen und erst in habsburgischer Zeit ins «Dreieck» im Kluseneingang verlegt worden. Als Begründung wird angeführt, daß ursprünglich der Weg nicht durch die Klus, sondern östlich des Burgfelsens über das sogenannte "Högerli» geführt habe. 7 Landeskarte 1:25 000, Blatt 1088 Hauenstein und Geologisch-Morphologische Karte in Lit. 24. 8 Heute ist der Unterschied durch kraftwerkbedingten Aufstau der Aare allerdings geringer.

9 Landeskarte 1:25 000, Blatt 1108 Murgenthal, und Lageskizzen in Lit. 6.

10 Landeskarte 1:25000, Blatt 1108 Murgenthal und Geologisch-Morphologische Karte in Lit. 22.

11 Landeskarte 1:25 000, Blatt 1068 Sissach und Geologische Karte in Lit. 26. Eine Lageskizze findet sich ferner in Lit. 4.

12 Landeskarte 1:25000, Blatt 1088 Hauenstein und Geologische Karte in Lit. 27.

13 Landeskarte 1: 25 000, Blatt 1107 Balsthal und Morphologische Karte in Lit. 2.

14 Landeskarte $1: 25$ 000, Blatt 1108 Murgenthal und Geologische Karte in Lit. 28.

15 Landeskarte 1:25 000, Blatt 1109 Schöftland und Geologische Karte in Lit. 29.

16 Unsere Eintragungen basieren in erster Linie auf Angaben in Lit. 1 und 21. Die froburgische Stadtrechtsfamilie wird in Lit. 21 untersucht. Darin wird u. a. festgestellt, daß über das Privatrecht von Liestal, Waldenburg, Wiedlisbach, Falkenstein und Fridau nichts bekannt ist; für Wiedlisbach, Fridau und Falkenstein fehlen überhaupt die Stadtrechte, und aus den Rechten von Liestal und Waldenburg lassen sich seit der Herrschaft der Stadt Basel keine zuverlässigen Anhaltspunkte über die originäre Entwicklung der beiden Städte mehr gewinnen.

17 Zunahme 1950-1960: Waldenburg 4,9\%, Oberdorf $19,1 \%$, Niederdorf $38,2 \%$, Hölstein $24,0 \%$.

18 Wiedlisbach hat jedoch zusammen mit Wangen a. A. einen Autobahnanschluß bekommen, nach denjenigen von Oensingen und Niederbipp übrigens der dritte auf einer Autobahnstrecke von nur $7 \mathrm{~km}$. 19 Es besteht allerdings ein Projekt, die Berner Linie von Rothrist her auf dem linken, westlichen Aareufer nach Olten zu führen, womit die Gabelung in Aarburg wegfiele.

\section{Résumé}

Au tournant des 12 ième et 13 ième siècles, les comtes de Froburg fondèrent 8 villes aux abords des deux cols du Hauenstein: Liestal, Wạldenburg, Olten, Falkenstein/Klus, Aarburg, Zofingue, Wiedlis- 
bach et Fridau. Caractérisées par leur plan axial et leur petite surface variant de 8 à 0,5 ha, toutes ces villes remplissaient une fonction purement militaire. Elles se trouvaient trop près les unes des autres et lors de leur fondation, on n'avait nullement tenu compte des données économiques. C'est pourquoi aucune de ces villes ne put se développer avant le 19 ième siècle. Elles demeurèrent de petits bourgs campagnards; Fridau disparut même à la suite de l'invasion des Gugler en 1375 et Falkenstein/Klus dépérit peu à peu.

Ce n'est qu'au cours du 19 ième siècle que les villes de Liestal et d'Olten prirent leur essor, celle-ci, grâce à sa qualité de nœud ferroviaire, celle-là en rapport avec sa fonction de chef-lieu de canton. Le développement du trafic ferroviaire amena à Olten de multiples industries qui, au 20 ième siècle, par manque d'espace, débordèrent vers les villes voisines d'Aarburg et de Zofingue, devenues aujourd'hui d'importants centres industriels. Olten et Zofingue disposent actuellement d'une importante banlieue tandis que moins favorisés par leur situation géographique, les bourgs de Wiedlisbach et de Waldenburg ne se sont guère développés. Olten, Aarburg et Zofingue forment aujourd'hui une agglomération d'environ 90000 habitants. Cette situation rend particulièrement difficiles les problèmes d'aménagement régional, d'autant plus que la zone est coupée en deux par la frontière cantonale. Grâce à leur rayonnement, Olten, Liestal et Zofingue ont aussi modifié considérablement la structure des villages environnants. 